Draft Version December 23, 2020

Typeset using LATEX default style in AASTeX62

\title{
ANALYTIC STABILITY MAPS OF UNKNOWN EXOPLANET COMPANIONS FOR IMAGING PRIORITIZATION
}

\author{
Carlos Gascon, ${ }^{1,2}$ Dmitry Savransky,${ }^{3,4}$ and Miquel Sureda ${ }^{1}$ \\ ${ }^{1}$ ESEIAAT, Polytechnic University of Catalonia, Terrassa, Barcelona 08222, Spain \\ ${ }^{2}$ School of Mathematics and Statistics, Polytechnic University of Catalonia, Barcelona, Barcelona 08028, Spain \\ ${ }^{3}$ Sibley School of Mechanical and Aerospace Engineering, Cornell University, Ithaca, NY 14853, USA \\ ${ }^{4}$ Carl Sagan Institute, Cornell University, Ithaca, NY 14853, USA
}

\begin{abstract}
Identifying which systems are more likely to host an imageable planet can play an important role in the construction of an optimized target list for future direct imaging missions, such as the planned Coronagraph Instrument (CGI) technology demonstration for the Nancy Grace Roman Space Telescope. For single-planet systems, the presence of an already detected exoplanet can severely restrict the target's stable region and should therefore be considered when searching for unknown companions. To do so, we first analyze the performance and robustness of several two-planet stability criteria by comparing them with long-term numerical simulations. We then derive the necessary formulation for the computation of $(a, R)$ analytic stability maps, which can be used in conjunction with depth-of-search grids in order to define the stable-imageable region of a system. The dynamically stable completeness (i.e., the expected number of imageable and stable planets) can then be calculated via convolution with the selected occurrence grid, obtaining a metric that can be directly compared for imaging prioritization. Applying this procedure to all the currently known single-planet systems within a distance of $50 \mathrm{pc}$, we construct a ranked target list based on the CGI's predicted performance and SAG13 occurrence rates. Finally, we evaluate the importance of considering the radial velocity data from past Doppler surveys in order to rule out entire regions of our parameter space where, if a planet existed, it would have certainly been detected by previous RV observations.
\end{abstract}

Keywords: methods: analytical - planets and satellites: dynamical evolution and stability - planets and satellites: detection

\section{INTRODUCTION}

While indirect detection methods, such as radial velocity and transit photometry, have been the main source of exoplanetary information to date, direct imaging has emerged as a challenging but highly desirable technique, providing unique information regarding the atmospheric structure and chemical composition of exoplanets (Konopacky et al. 2013). In this context, space-based direct imaging instruments, such as the Nancy Grace Roman Space Telescope's Coronagraph Instrument (CGI), will enable the expansion and better characterization of the known population of exoplanets. Given the high cost and complexity of space observatories, detailed and extensive planning is required in order to ensure the successful execution of the mission. In particular it is essential to create optimized target lists by identifying which systems are more likely to host imageable planets.

Garrett et al. (2017) addressed this problem by defining the depth-of-search grids in the $(a, R)$ space, where the value of each bin represented the probability of detecting a planet with semi-major axis $a$ and radius $R$. The resultant imageable region was obtained according only to the instrument's performance and capabilities, allowing for the estimation of the expected number of detected planets (i.e., total completeness) by convolution with an assumed grid of occurrence rates. A similar procedure can be used to evaluate the observability of previously discovered planets. This provides two potential sources of targets: stars with no known planets where a new planet is likely to be observable, and stars with known planets that could potentially be imaged with a new instrument. There remains, however, a third category: stars with known planets that are not observable with proposed instrumentation, but which may harbor additional planets that could be imaged. There also exists an overlapping population between the second and third 
categories, composed of targets where the known planet could be imaged, but we would also be interested in detecting additional, unknown, companions via imaging.

When searching for additional exoplanets in already known single-planet systems, the gravitational effect of the existing body can severely restrict the target's stable region and must therefore be taken into account. For instance, let us consider a nearby star with a large imageable region and a massive highly eccentric planet in the center of such a region. In this context, most of the detectable area would be chaotic due to the known planet's presence, consequently reducing the probability of detecting a stable unknown companion in a system which a priori seemed a valuable target. In general, for any system the following question is naturally raised: How many stable unknown companions could an instrument detect? We seek to answer this question in an accurate but computationally inexpensive manner, in order to rapidly identify which targets have a higher probability of hosting an additional planet and discard those systems where no unknown companions could be detected. To do so, in Section 2 we begin by describing several two-planet stability criteria and comparing them with long-term numerical simulations. In Section 3 we derive the necessary expressions for the conditional density function of the outer pericenter to inner apocenter ratio $(\rho)$ and the angular momentum deficit $(C)$, which will be essential for the computation of analytic stability maps presented in Section 4.

Finally, in Section 5 we make use of these results, together with depth-of-search and occurrence grids, in order to obtain the expected number of imageable and stable planets for a given target. We conclude by presenting 213 currently known single-planet systems within 50 parsecs of the Earth, ranked according to the CGI's predicted performance and the Study Analysis Group 13 (SAG13) occurrence rates. This ranking, when coupled with updated Doppler sensitivities for potential targets, could aide researchers working on any CGI participating science program that follows the initial technology demonstration phase of the instrument, and would potentially greatly expand the sample of targets observed by both Doppler spectroscopy and direct imaging.

\section{ANALYTIC STABILITY CRITERIA FOR TWO-PLANET SYSTEMS}

Unlike systems with three or more planets, the stability of two-planet systems can be analytically characterized via several different criteria. One of the main results was obtained by Marchal \& Bozis (1982), who extended the notion of Hill stability to the general three-body problem and showed that certain initial conditions can preclude close encounters between the outer planet and the inner bodies. Based on this result, Gladman (1993) found that two planets in initially circular and coplanar orbits are Hill stable if

$$
a_{2}-a_{1}>2 \sqrt{3} R_{H}
$$

where

$$
R_{H}=\left(\frac{m_{1}+m_{2}}{3 M_{\star}}\right)^{1 / 3} \frac{a_{1}+a_{2}}{2}
$$

is the mutual Hill radius, $M_{\star}$ is the mass of the central star, $a_{i}$ are the semi-major axes of the planet orbits, $m_{i}$ are the planetary masses, and the subscripts 1 and 2 refer to the inner and outer planet, respectively. It is important to note, however, that long-term interactions between planets in Hill stable orbits could still ultimately lead to the ejection of the outer planet or the collision of the inner planet with the star (i.e., Lagrange instability). From another perspective, Wisdom (1980) applied the resonance overlap criterion to the coplanar and circular restricted three body problem. By studying the region around a planet where first order mean motion resonances (MMRs) overlap, the author found that a test particle with semi-major axis $a$ would experience chaotic motion if

$$
\frac{\left|a-a_{p}\right|}{a_{p}}<C_{\mathrm{w}} \mu_{p}^{2 / 7}
$$

where $C_{\mathrm{w}}$ is a constant value, $a_{p}$ is the semi-major axis of the planet and $\mu_{p}=m_{p} / M_{\star}$ is the mass ratio between the planet and the star. Although originally Wisdom obtained a theoretical value of $C_{\mathrm{w}}=1.33$, Duncan et al. (1989) presented a numerically-derived estimate of $C_{\mathrm{d}}=1.57$. For the case of two massive planets in circular orbits, Deck et al. (2013) extended Wisdom's criterion and predicted that all orbits should be chaotic if

$$
\frac{a_{2}-a_{1}}{a_{1}}<1.46 \epsilon^{2 / 7}
$$


where $\epsilon=\left(m_{1}+m_{2}\right) / M_{\star}$ is the planets-to-star mass ratio. Deck et al. (2013) also developed a similar expression for non-circular configurations, only applicable, however, when the weighted eccentricity satisfies $\sigma \approx e_{1}+e_{2} \gtrsim 1.33 \epsilon^{3 / 7}$ and eccentricities are low enough that overlap of resonances of second order and beyond are negligible. For arbitrary eccentricities, several criteria have been proposed (Giuppone et al. 2013; Petrovich 2015; Laskar \& Petit 2017; Petit et al. 2017; Petit et al. 2018), which we will divide into two generic categories depending on whether the criterion is based on the outer pericenter to inner apocenter ratio $(\rho)$ or the angular momentum deficit $(\boldsymbol{C})$.

\subsection{Criteria based on the Outer Pericenter to Inner Apocenter ratio}

As demonstrated by Petrovich (2015), most of the proposed two-planet stability criteria for arbitrary eccentricities can be expressed as a boundary of the ratio between the pericenter of the outer planet and the apocenter of the inner planet, here denoted by

$$
\rho=\frac{a_{2}\left(1-e_{2}\right)}{a_{1}\left(1+e_{1}\right)}
$$

where $e_{1}$ and $e_{2}$ are the corresponding eccentricities. Giuppone et al. (2013) developed an extended crossing orbit criterion by adding and subtracting Wisdom's overlap region (Wisdom 1980, Equation 3) to the outer pericentric and inner apocentric distances, respectively. They also took into account the effect of the difference in the longitudes of the pericenter $\Delta \bar{\omega}$ and proposed stability limits for the case of aligned $\left(\Delta \bar{\omega}=0^{\circ}\right)$ and anti-aligned $\left(\Delta \bar{\omega}=180^{\circ}\right)$ initial orbits. By studying the stability limits of a test planet around a known and existing planet, they presented the following criterion for the anti-aligned configuration

$$
\rho>\left\{\begin{array}{cc}
\frac{1}{1-\delta} & a_{2}=a_{k} \\
1+\delta & a_{1}=a_{k},
\end{array}\right.
$$

where $a_{k}$ is the known planet's semi-major axis and $\delta=1.57\left(\mu_{1}^{2 / 7}+\mu_{2}^{2 / 7}\right)$. Here we will make use of the modification proposed by Hadden \& Lithwick (2018), where they employ $\delta=1.46 \epsilon^{2 / 7}$ in accordance to the results of Deck et al. (2013). Alternatively, Petrovich (2015) approached the problem numerically by performing long-term integrations for a large number of planetary systems and a wide range of eccentricities and inclinations. They found $\rho$ to be the single parameter that best described the stability boundary and presented the following empirical criterion

$$
\rho>1.15+2.4\left[\max \left(\mu_{1}, \mu_{2}\right)^{1 / 3}\right]\left(\frac{a_{2}}{a_{1}}\right)^{1 / 2} .
$$

\subsection{Criteria based on the Angular Momentum Deficit}

Considering the secular approximation of a planetary system, Laskar \& Petit (2017) developed an alternative stability criterion based on the conservation of the angular momentum deficit (AMD). Following the definition of Laskar (2000), the AMD $(C)$ is given by the difference between the norm of the angular momentum of an equivalent circular and coplanar system and the norm of the real system's angular momentum, which for a system of $n_{p}$ planets is

$$
C=\sum_{j=1}^{n_{p}} \Lambda_{j}\left(1-\sqrt{1-e_{j}^{2}} \cos i_{j}\right),
$$

where $i_{j}$ is the relative inclination, $\Lambda_{j}=m_{j} \sqrt{G M_{\star} a_{j}}$ and $G$ is the gravitational constant. For a two-planet system, Laskar \& Petit (2017) defined the relative angular momentum deficit as

$$
\mathscr{C}=\frac{C}{\Lambda_{2}}=\gamma \sqrt{\alpha}\left(1-\sqrt{1-e_{1}^{2}} \cos i_{1}\right)+\left(1-\sqrt{1-e_{2}^{2}} \cos i_{2}\right)
$$

where $\alpha=a_{1} / a_{2}$ represents the semi-major axis ratio and $\gamma=m_{1} / m_{2}$ is the mass ratio. In this context, they obtained the minimum relative AMD which allowed for planetary collisions, referred to as the collisional critical AMD $\left(C_{c}^{\mathrm{C}}\right)$. Consequently, since the AMD is conserved at all orders (Laskar \& Petit 2017), the impossibility of collisions between the two planets is ensured if the initial relative AMD is bounded as

$$
\mathscr{C}<C_{c}^{\mathrm{C}}(\alpha, \gamma)
$$


This condition can be extended to multiple planet systems by analyzing the AMD-stability of every pair of adjacent planets, as well as the innermost planet and the star. Furthermore, Agnew et al. (2018) compared the previous criterion with numerical simulations over a large number of known systems and concluded that the AMD-stability is a reliable tool for determining the stability of planetary systems. In order to take into account the effect of mean motion resonances (MMR) ignored by the secular theory, Petit et al. (2017) proposed a new derivation of the first-order MMR overlap criterion in the AMD framework. They refined the criteria presented by Wisdom (1980) and Deck et al. (2013) by deriving a more global expression, for which they then associated a new critical AMD $\left(C_{c}^{\mathrm{MMR}}\right)$. Since it only makes sense to apply the first-order MMR criterion when $\alpha$ is close to 1 , they combined this with the previous collision criterion $\left(C_{c}^{\mathrm{C}}\right)$ and defined the following piece-wise critical AMD (Petit et al. 2017)

$$
\mathscr{C}<C_{c}(\alpha, \gamma, \epsilon)= \begin{cases}C_{c}^{\mathrm{C}}(\alpha, \gamma) & \alpha<\alpha_{R}(\epsilon, \gamma) \\ C_{c}^{\mathrm{MMR}}(\alpha, \gamma, \epsilon) & \alpha>\alpha_{R}(\epsilon, \gamma)\end{cases}
$$

where $\alpha_{R}$ represents the semi-major axis ratio at which $C_{c}^{\mathrm{C}}=C_{c}^{\mathrm{MMR}}$. For lower values of $\alpha$, the collisional criterion becomes stricter and consequently more useful.

Continuing their work in the AMD framework, Petit et al. (2018) generalized the stability criterion proposed by Gladman (1993) and defined the Hill stability AMD criterion

$$
\mathscr{C}<C_{c}^{\mathrm{H}}(\alpha, \gamma, \epsilon)=\gamma \sqrt{\alpha}+1-(1+\gamma)^{3 / 2} \sqrt{\frac{\alpha}{\gamma+\alpha}\left(1+\frac{3^{4 / 3} \epsilon^{2 / 3} \gamma}{(1+\gamma)^{2}}\right)}
$$

where $C_{c}^{\mathrm{H}}$ is defined as the Hill critical AMD. As this expression was obtained as an approximation of the criterion from Marchal \& Bozis (1982), Petit et al. (2018) compared both criteria and proved that Equation (12) is accurate for the typical range of values of $\epsilon$ and still valid for very large or small planetary mass ratios $(\gamma)$.

\subsection{Numerical Simulations and Criteria Comparison}

To assess the performance and robustness of the criteria described above, we performed several numerical simulations in order to study and compare their behaviour over a wide range of parameters. Specifically, we added a test planet to two known single-planet systems (HD 154345 and HD 114613) and analyzed the long-term stability of the resultant two-planet systems. The stellar parameters and the orbital elements of the known planet, denoted by the subscript $k$, were extracted from the NASA Exoplanet Archive ${ }^{1}$ and can be found summarized in Table 1 . For simplicity, all systems were assumed to be coplanar and the existing planet's mass was considered to be the minimum value $m_{k}$ sin $I$, where $I$ is the system's inclination with respect to the line of sight. The remaining unknown parameters, such as the longitude of the ascending node or the initial mean anomaly, were all set to zero. For each system, we then constructed two different types of stability maps.

1. (a, e) stability map: regular grid with 70 logarithmically spaced semi-major axis bins and 40 linearly spaced eccentricity bins. The ranges of $a$ and $e$ were selected taking into the account the extension of the chaotic region around the known planet's semi-major axis $a_{k}$. The test planet's mass $m$ was constant through the whole grid, being fixed in a different value depending on the system. In particular, for the system HD 114613 a mass of 1 $M_{\mathrm{J}}$ was employed, while a smaller value was used in the case of HD 154345 . On the other hand, the argument of periastron $\omega \in[0,2 \pi]$ was always randomly generated.

2. (a, m) stability map: regular grid with 70 semi-major axis bins and 40 mass bins, both logarithmically spaced. The range of values of $a$ and $m$ was determined considering the imageable region of the system with the CGI. In this case, the test planet's eccentricity $e$ was constant through the whole grid, being fixed in a different value depending on the system. In the case of the system HD 154345, a nearly circular value was used, while a higher eccentricity was assigned to the test planet in the system HD 114613. Finally, $\omega$ was again randomly generated.

The particular values employed for every system and stability map are presented in Table 1 . For each bin, we integrated the corresponding two-planet system using the Leapfrog integrator implemented in the REBOUND package

\footnotetext{
1 The required orbital parameters where retrieved from the NASA Exoplanet Archive (https://exoplanetarchive.ipac.caltech.edu) on 2019 June 8.
} 
Table 1. Stellar and Planetary Parameters

\begin{tabular}{|c|c|c|c|c|c|c|c|c|c|c|c|c|}
\hline \multicolumn{3}{|c|}{ Target Star } & \multicolumn{4}{|c|}{ Known Planet } & \multicolumn{3}{|c|}{$(a, e)$ map } & \multicolumn{3}{|c|}{$(a, m)$ map } \\
\hline Name & $\begin{array}{c}\text { Distance } \\
\text { (pc) }\end{array}$ & $\begin{array}{c}M_{\star} \\
\left(M_{\odot}\right)\end{array}$ & $\begin{array}{c}a_{k} \\
(\mathrm{AU})\end{array}$ & $e_{k}$ & $\begin{array}{c}m_{k} \sin I \\
\left(M_{\mathrm{J}}\right)\end{array}$ & $\begin{array}{l}\omega_{k} \\
\left(^{\circ}\right)\end{array}$ & $\begin{array}{c}a \\
(\mathrm{AU})\end{array}$ & $e$ & $\begin{array}{c}m \\
\left(M_{\mathrm{J}}\right)\end{array}$ & $\begin{array}{c}a \\
(\mathrm{AU})\end{array}$ & $e$ & $\begin{array}{c}m \\
\left(M_{\mathrm{J}}\right)\end{array}$ \\
\hline HD 154345 & 18.294 & 0.71 & 4.21 & 0.04 & 0.82 & $0^{\mathrm{a}}$ & {$[2,12]$} & {$[0,0.5]$} & 0.1 & {$[1.5,25]$} & 0.05 & {$[0.067,134.45]$} \\
\hline HD 114613 & 20.295 & 1.27 & 5.34 & 0.458 & 0.357 & 196 & {$[1.5,20]$} & {$[0,0.5]$} & 1 & {$[1.5,25]$} & 0.1 & {$[0.067,134.45]$} \\
\hline
\end{tabular}

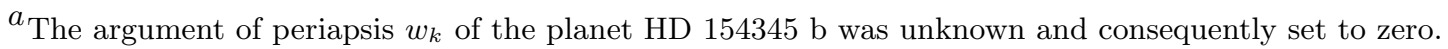

Note-The $(a, m)$ grid was ranged taking into account the imageable region of each system, which was assumed to be approximately the same in both cases. In particular, the limiting values of $m$ correspond to a planetary radius $R$ between 4 and $17 R_{\oplus}$.

(Rein \& Liu 2012). The simulations were run for $10^{9} \mathrm{yr}$ with a timestep of $T_{1} / 50$, where $T_{1}$ is the orbital period of the innermost planet. Integrations were terminated if the two planets approached one another within one mutual Hill radius (Equation 2), or if a planet reached an astrocentric distance of either $5 \times 10^{-3}$ or $250 \mathrm{AU}$.

Figure 1 shows the resultant stability maps for a nearly circular configuration (HD 154345) and a highly eccentric system (HD 114613). In general, we observe that Petrovich's criterion shows the most conservative boundaries, appearing to be too pessimistic in the first case and slightly more appropriate for large eccentricities. In contrast, the complete AMD stability limit (Equation 11) falls inside the chaotic region in most cases and will be consequently discarded for the purposes of the following sections. Similarly to Giuppone's criterion, the Hill AMD boundaries offer an acceptable necessary condition for stability and could therefore be used as a more optimistic alternative. In particular, we remark how the Hill AMD stability condition accurately delimits the earliest chaotic orbits in both $(a$, e) maps, likely corresponding to the region where planetary close encounters occur. Hence, the remaining instabilities outside these boundaries may be the result of ejections or collisions between the inner planet and the star, which by definition are not taken into account in the Hill criterion. Furthermore, the HD $114613(a, m)$ stability map shows a pronounced increase in the Hill stability limits as the test planet's mass decreases, disagreeing with other criteria and the numerical simulations. Such behavior, which essentially increases the predicted unstable region, becomes more significant for high eccentricities and can be related to the Hill stability's strong dependence on the planetary mass ratio $\gamma$ for non-circular configurations (Deck et al. 2013). We must keep in mind that the Hill stability criterion from Marchal \& Bozis (1982) cannot be directly applied to the elliptic restricted three body problem and therefore should not be used when one of the planetary masses is close to zero. Nonetheless, given that in most cases the CGI-imageable region only covers high-mass planets, the Hill AMD can still be considered a valid criterion in the following sections. Regarding the test planet's mean motion resonances with the existing planet, we note how for the nearly circular case, the stable resonant lines are more predominant and extend up to larger values of $e$ and $m$, while being less numerous and significant in the high-eccentricity system.

\section{DERIVATIONS}

Let us consider a coplanar, three-body system consisting of a central star of mass $M_{\star}$, and two orbiting planets, where the mass and orbital elements of one of the planets $\left(a_{k}, e_{k}, m_{k}\right)$ are known. The remaining planet is unknown, and its parameters $(a, e$ and $m$ ) will be consequently treated as random variables. In particular, the eccentricity $e$ will follow a Rayleigh distribution with parameter $\sigma$ (i.e. mean eccentricity $\mu_{e}=\sigma \sqrt{\pi / 2}$ ), while the semi-major axis $a$ and the planet's mass $m$ will have a joint probability density function $f_{\bar{a}, \bar{m}}(a, m)$ representative of the population of interest. With this setup, for fixed values of $a$ and $m$, the integral of the conditional density function of both the AMD $(C)$ and the outer pericenter and inner pericenter ratio $(\rho)$ can be easily solved. As will be shown in Section 4 , this result will be essential for the computation of the analytic stability maps and the prioritization of planetary systems for followup imaging.

\section{1. $\rho$ Conditional Density Function}



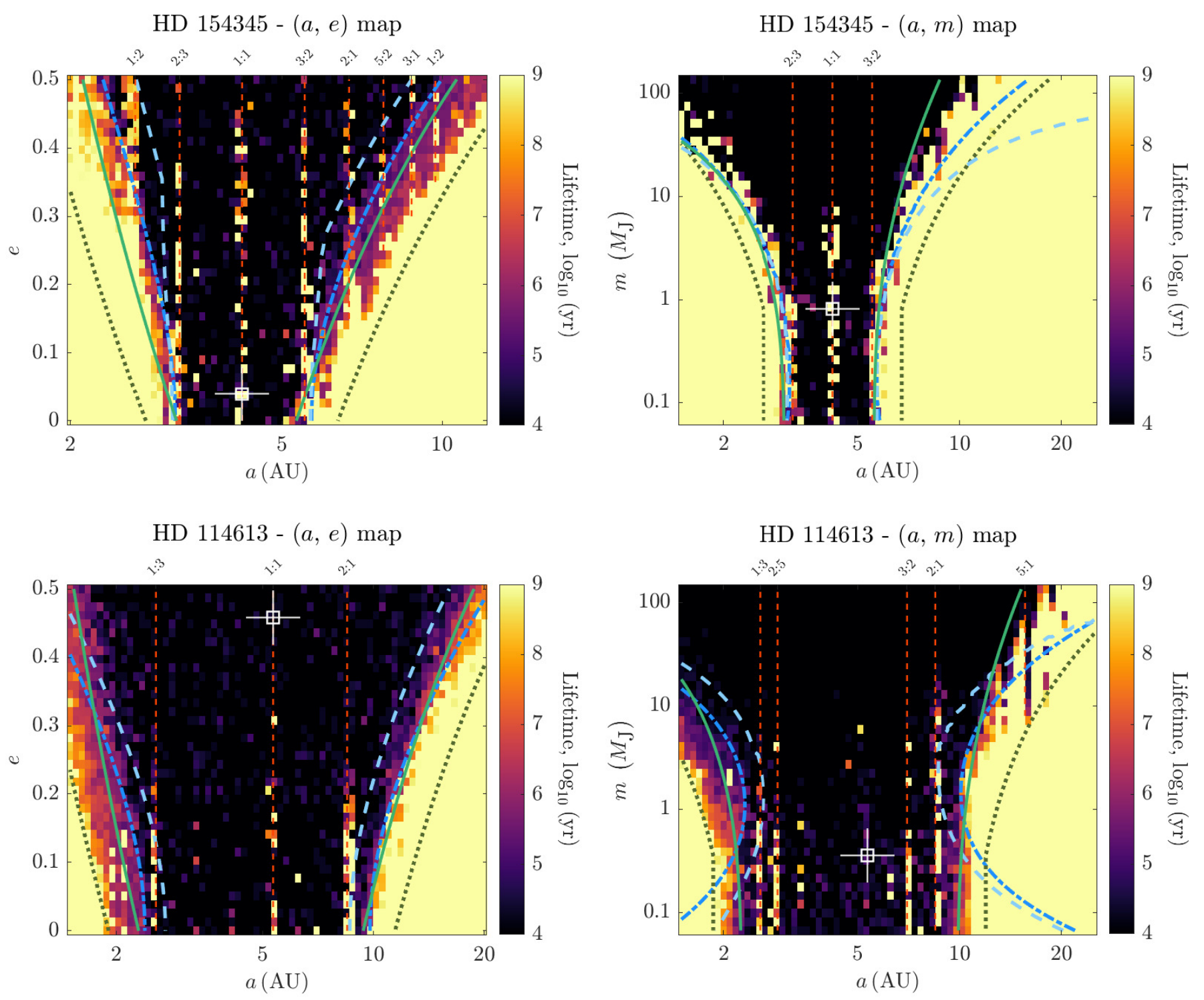

---- Hill AMD Crit. ....... Petrovich's Crit. - - Complete AMD Crit. —— Giuppone's Modified Crit.

Figure 1. Numerical stability maps for the nearly circular system HD 154345 (top) and high eccentricity system HD 114613 (bottom), compared to the stability boundaries given by the criteria specified in the legend. The white marker indicates the position of the existing planet and the red dashed lines indicate low-order mean motion resonances with the known planet.

Making use of Equation (5), the outer pericenter to inner apocenter ratio can be rewritten as

$$
\rho=g_{\rho}(e, a)= \begin{cases}\frac{q_{k}}{a(1+e)} & a<a_{k} \\ \frac{a(1-e)}{Q_{k}} & a>a_{k},\end{cases}
$$

where $q_{k}=a_{k}\left(1-e_{k}\right)$ and $Q_{k}=a_{k}\left(1+e_{k}\right)$ are, respectively, the known planet's pericenter and apocenter. For a fixed semi-major axis $a$, we observe that $\rho=g_{\rho}(e \mid a)$ is a univariate function only dependent on $e$. Consequently, the inverse function $h_{\rho}=g_{\rho}^{-1}(\rho \mid a)$ is directly obtained by isolating the eccentricity in Equation (13)

$$
e=h_{\rho}(\rho \mid a)= \begin{cases}\frac{q_{k}-a \rho}{a \rho} & a<a_{k} \\ \frac{a-Q_{k} \rho}{a} & a>a_{k} .\end{cases}
$$


For simplicity, we will omit the conditional notation in $h_{\rho}(\rho)$ from here on out, since we are primarily interested in evaluating all expressions for a given value of $a$. Taking the derivative of Equation (14) with respect to $\rho$, we get

$$
\left|\frac{d h_{\rho}}{d \rho}(\rho)\right|= \begin{cases}\frac{q_{k}}{a \rho^{2}} & a<a_{k} \\ \frac{Q_{k}}{a} & a>a_{k} .\end{cases}
$$

Using Equations (14) and (15), the $\rho$ conditional density function is then given by

$$
f_{\bar{\rho} \mid \bar{a}}(\rho \mid a)=f_{\bar{e}}\left(h_{\rho}(\rho)\right)\left|\frac{d h_{\rho}}{d \rho}(\rho)\right|
$$

where $f_{\bar{e}}(e)$ is the density function of the eccentricity, assumed to be Rayleigh distributed. For fixed values of $a$ and $m$, the probability of having a stable configuration, denoted by $S_{\rho}(a, m)$, is obtained by integrating Equation (16) over the region defined by the specific $\rho$ stability criterion used:

$$
S_{\rho}(a, m)=\int_{\rho_{c, l}}^{\rho_{c, u}} f_{\bar{\rho} \mid \bar{a}}(\rho \mid a) d \rho=F_{\bar{e}}\left(h_{\rho}\left(\rho_{c, u}\right)\right)-F_{\bar{e}}\left(h_{\rho}\left(\rho_{c, l}\right)\right),
$$

where $\rho_{c, l}(a, m)$ and $\rho_{c, u}(a, m)$ are the lower and upper limit, respectively. Given our assumptions, the integral can be simply calculated as the difference between the Rayleigh cumulative distribution function $F_{\bar{e}}(e)$ evaluated at the limiting eccentricities $h_{\rho}\left(\rho_{c, u}\right)$ and $h_{\rho}\left(\rho_{c, l}\right)$. Substituting $h_{\rho}$ from Equation (14) yields the solution

$$
S_{\rho}(a, m)=\left\{\begin{array}{l}
-\left.\exp \left(\frac{-1}{2 \sigma^{2}}\left(\frac{q_{k}-a \rho}{a \rho}\right)^{2}\right)\right|_{\rho_{c, l}} ^{\rho_{c, u}} \quad a<a_{k} \\
-\left.\exp \left(\frac{-1}{2 \sigma^{2}}\left(\frac{a-Q_{k} \rho}{a}\right)^{2}\right)\right|_{\rho_{c, l}} ^{\rho_{c, u}} \quad a>a_{k} .
\end{array}\right.
$$

The majority of currently available exoplanet data for CGI-imageable planets has been obtained from radial velocity (RV) surveys. While transit photometry currently leads in the total number of exoplanet discoveries, most of these (primarily due to Kepler and the K2 mission) are too distant for imaging with the next generation of space-based coronagraphic instruments, and the CGI in particular. While we expect this to change with the Transiting Exoplanet Survey Satellite (TESS) and other surveys, for now, the true mass $m_{k}$ of the majority of known exoplanets of interest remains undetermined, and only the minimum mass $m_{k, \min }=m_{k} \sin I$ is known. In these cases, we rewrite the probability integral (17) as $S_{\rho}\left(a, m, m_{k}\right)$ and we introduce the system's inclination $I \in[0, \pi)$ as a new random variable with a sinusoidal probability density function $f_{\bar{I}}(I)=\sin (I) / 2$. We can then take into account the effect of the known planet's mass uncertainty by using Equation (18) and defining

$$
S_{\rho}^{\prime}(a, m)=\int_{0}^{\pi} S_{\rho}\left(a, m, \frac{m_{k, m i n}}{\sin (I)}\right) f_{\bar{I}}(I) \mathrm{d} I .
$$

For the stability criteria based on $\rho$, Equations (18) and (19) will be directly used for the computation of analytic stability maps. Similarly, we now derive the equivalent formulation for the angular momentum deficit $C$.

\subsection{Conditional Density Function}

For a two-planet system $\left(n_{p}=2\right)$, Equation (8) can be written as

$$
C=g_{C}(e, a, m)=K+\Lambda\left(1-\sqrt{1-e^{2}}\right),
$$

where $K=\Lambda_{k}\left(1-\sqrt{1-e_{k}^{2}}\right)$ accounts for the known planet AMD contribution. For fixed values of $a$ and $m, \Lambda=$ $m \sqrt{G M_{\star} a}$ is completely defined and therefore $C=g_{C}(e \mid a, m)$ is only a function of $e$. The inverse function $g_{C}^{-1}(C \mid$ $a, m)$, which we will denote as $h_{C}$, is then obtained by solving Equation (20) for the eccentricity

$$
e=h_{C}(C \mid a, m)=\sqrt{1-\left(\frac{\Lambda-C+K}{\Lambda}\right)^{2}},
$$


where again the conditional notation will be dropped for simplicity. Since the inverse function must have the range $e=h_{C}(C) \in[0,1)$, the derivative

$$
\left|\frac{d h_{C}}{d C}(C)\right|=\frac{1}{\Lambda}\left(\frac{\sqrt{1-h_{C}^{2}(C)}}{h_{C}(C)}\right)
$$

is well defined except for the case $C=K$ (i.e. $h_{C}(C)=0$ ). However, this singularity is naturally solved when the expression of the conditional density function is simplified

$$
f_{\bar{C} \mid \bar{a}, \bar{m}}(C \mid a, m)=f_{\bar{e}}\left(h_{C}(C)\right)\left|\frac{d h_{C}}{d C}(C)\right|=\frac{\sqrt{1-h_{C}^{2}(C)}}{\Lambda \sigma^{2}} \exp \left(\frac{-h_{C}^{2}(C)}{2 \sigma^{2}}\right),
$$

where the formula of the Rayleigh probability density function for $f_{\bar{e}}(e)$ has been used. Following the same procedure as in Section 3.1, for certain values of $C_{c, l}(a, m)$ and $C_{c, u}(a, m)$ determined by the specific AMD stability criterion used, the integral of the conditional density function is

$$
S_{C}(a, m)=\int_{C_{c, l}}^{C_{c, u}} f_{\bar{C} \mid \bar{a}, \bar{m}}(C \mid a, m) \mathrm{d} C=F_{\bar{e}}\left(h_{C}\left(C_{c, u}\right)\right)-F_{\bar{e}}\left(h_{C}\left(C_{c, l}\right)\right) .
$$

Making use of Equation (21), the analytic solution is then given by

$$
S_{C}(a, m)=-\left.\exp \left(\frac{1}{2 \sigma^{2}}\left(\left(\frac{\Lambda-C+K}{\Lambda}\right)^{2}-1\right)\right)\right|_{C_{c, l}} ^{C_{c, u}}
$$

As described in the previous subsection, in the case of systems with an undetermined planetary mass $m_{k}$, we redefine the probability of having a stable configuration as

$$
S_{C}^{\prime}(a, m)=\int_{0}^{\pi} S_{C}\left(a, m, \frac{m_{k, m i n}}{\sin (I)}\right) f_{\bar{I}}(I) \mathrm{d} I .
$$

Although a more detailed and consistent single-planet ranking is described in Section 5, a first approach relies on the definition of the $a, m$ and $C$ (or $\rho$ ) joint probability density function

$$
f_{\bar{a}, \bar{m}, \bar{C}}(a, m, C)=f_{\bar{a}, \bar{m}}(a, m) \cdot f_{\bar{C} \mid \bar{a}, \bar{m}}(C \mid a, m)
$$

By choosing the appropriate limits of integration which approximately define the imageable region, together with the stability boundaries of $C$, a rapid estimation of the probability of detecting a stable planet can be computed as

$$
\int_{m_{l}}^{m_{u}} \int_{a_{l}}^{a_{u}} f_{\bar{a}, \bar{m}}(a, m)\left(\int_{C_{c, l}}^{C_{c, u}} f_{\bar{C} \mid \bar{a}, \bar{m}}(C \mid a, m) d C\right) \mathrm{d} a \mathrm{~d} m
$$

where the term in the inner parentheses has already been analytically solved, simplifying the calculation to a double integral. Equation (28), which can be equivalently derived for $\rho$, can be used to discard those systems with barely any stable imageable region or to obtain a first imaging prioritization in a fast and computationally inexpensive manner.

\section{ANALYTIC STABILITY MAPS}

Following the previous assumptions and derivations, in this section we compute the analytic stability maps which will allow us to rapidly characterize the stable region of a particular single-planet system. Essentially, these maps consist of a regular grid with 100 semi-major axis bins and 100 mass bins, both logarithmically spaced and ranged around the system's imageable region. For a particular pair $(a, m)$, the value of the corresponding bin represents the probability of having a stable configuration according to the specific criterion used. For the systems where the known planet's mass $m_{k}$ is completely determined, the stability maps are built using Equations (18) and (25), depending on the type of criterion used. On the other hand, if only $m_{k} \sin I$ is known, Equations (19) and (26) are employed. To illustrate this, we select the empirical criterion presented by Petrovich (2015), since it appears to give the most conservative and 
consistent boundaries according to the results from Section 2.3. Based on the critical $\rho$ from inequality (7), we define the lower limit of integration

$$
\rho_{c, l}^{\mathrm{P}}(a, m)= \begin{cases}1.15+2.4\left[\max \left(\mu, \mu_{k}\right)^{1 / 3}\right]\left(a_{k} / a\right)^{1 / 2} & a<a_{k} \\ 1.15+2.4\left[\max \left(\mu_{k}, \mu\right)^{1 / 3}\right]\left(a / a_{k}\right)^{1 / 2} & a>a_{k} .\end{cases}
$$

In general, given that we are only considering elliptical orbits (i.e., $e \in[0,1)$ ), the outer pericenter to inner apocenter ratio must have a range $\rho \in\left(g_{\rho}(1, a), g_{\rho}(0, a)\right]$ and therefore, $\rho_{c, l}$ should always be adjusted to the range of values of $\rho$. That is, if $\rho_{c, l}(a, m)<g_{\rho}(1, a)$ then $\rho_{c, l}(a, m)=g_{\rho}(1, a)$, and equivalently if $\rho_{c, l}(a, m)>g_{\rho}(0, a)$ then $\rho_{c, l}(a, m)=$ $g_{\rho}(0, a)$. Furthermore, the expression of $g_{\rho}(0, a)$ allows us to set the upper limit as

$$
\rho_{c, u}(a)=g_{\rho}(0, a)= \begin{cases}\frac{q_{k}}{a} & a<a_{k} \\ \frac{a}{Q_{k}} & a>a_{k}\end{cases}
$$

In addition, we will also construct stability maps using Giuponne's criterion (Giuppone et al. 2013, Equation 6) and the Hill AMD criterion (Petit et al. 2018, Equation 12), which will serve as an alternative for ranking planetary systems. In the first case, the lower limit of integration is directly given by Equation (6) and can be written as

$$
\rho_{c, l}^{\mathrm{G}}(a, m)= \begin{cases}\frac{1}{1-1.46 \epsilon^{2 / 7}} & a<a_{k} \\ 1+1.46 \epsilon^{2 / 7} & a>a_{k},\end{cases}
$$

while the upper limit $\rho_{c, u}$ is again given by Equation (30). On the other hand, assuming that the stellar mass $M_{\star}$ is known, the Hill critical AMD is only a function of the semi-major axis and mass ratios. From the definition of $C_{c}^{\mathrm{H}}(\alpha, \gamma)$ given in Equation (12), we then derive the upper limit of integration as

$$
C_{c, u}(a, m)= \begin{cases}\Lambda_{k} C_{c}^{\mathrm{H}}\left(a / a_{k}, m / m_{k}\right) & a<a_{k} \\ \Lambda C_{c}^{\mathrm{H}}\left(a_{k} / a, m_{k} / m\right) & a>a_{k},\end{cases}
$$

for which again the range of values of $C \in\left[g_{C}(0, a, m), g_{C}(1, a, m)\right)$ should be taken into consideration. In particular, the lower limit of integration is defined and given by $C_{c, l}(a, m)=g_{C}(0, a, m)=K$.

We demonstrate this procedure by generating the $(a, m)$ analytic stability maps of the single-planet systems HD 154345 and HD 114613 (Figure 2). Since none of the system's inclinations are known, we make use of Equations (19) and (26), together with the limits of integration presented above. The mean eccentricity for the Rayleigh distribution is taken to be $\mu_{e}=0.225$ (Moorhead et al. 2011) and the required orbital parameters, as well as the range of values of $a$ and $m$, are as in Table 1. In the most favourable case where the unknown planet follows instead a circular orbit, the stable region would be delimited by the gray dashed curves in Figure 2, which essentially correspond to the $\mathbf{0}$ contour lines. Furthermore, we can easily build $(a, R)$ stability maps by considering instead a set of logarithmically spaced planetary radius $R$ bins and applying the previous expressions to the corresponding planetary masses. For each value of $R$, the related mass $m$ is predicted using the FORECASTER best-fit density model (Chen \& Kipping 2016), originally composed of linear segments (in log-log space) of the form

$$
R=10^{C+\log _{10}(m) S},
$$

where $C$ and $S$ are fit coefficients defined in four mass intervals: Terran, Neptunian, Jovian and Stellar Worlds. Due to inclusion of many tidally locked, inflated Jupiters in the model, the original results tend to overestimate the radius for Jovian-size planets, and so we slightly modify the initial fit by moderating the transition between the Saturn and Jupiter mass-radius points. Specifically, the Neptunian Worlds segment is adjusted to end at the Saturn mass-radius point, from which a new fit is added as a straight line (in the log-log space) until the Jupiter mass-radius point. Moreover, the Jovian segment is corrected to be a constant Jupiter radius value ranging from 1 Jupiter Mass through 0.08 Solar Masses. The Terran and Stellar Worlds, on the other hand, remain unchanged. The resultant values of $C$ and $S$, for Earth mass and radius units, are shown in Table 2. It is important to note that we are not suggesting that 
HD 154345 - Petrovich's Criterion

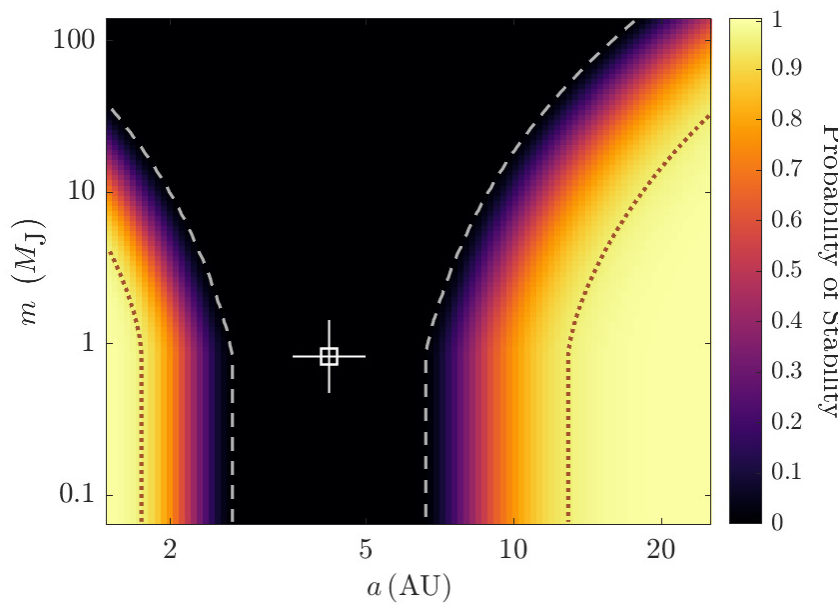

HD 154345 - Giuppone's Mod. Criterion

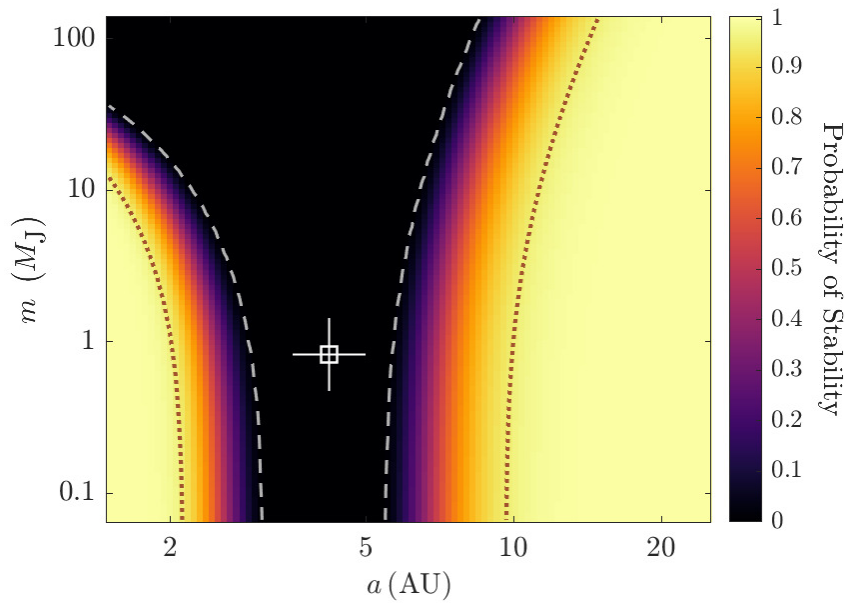

HD 154345 - Hill AMD Criterion

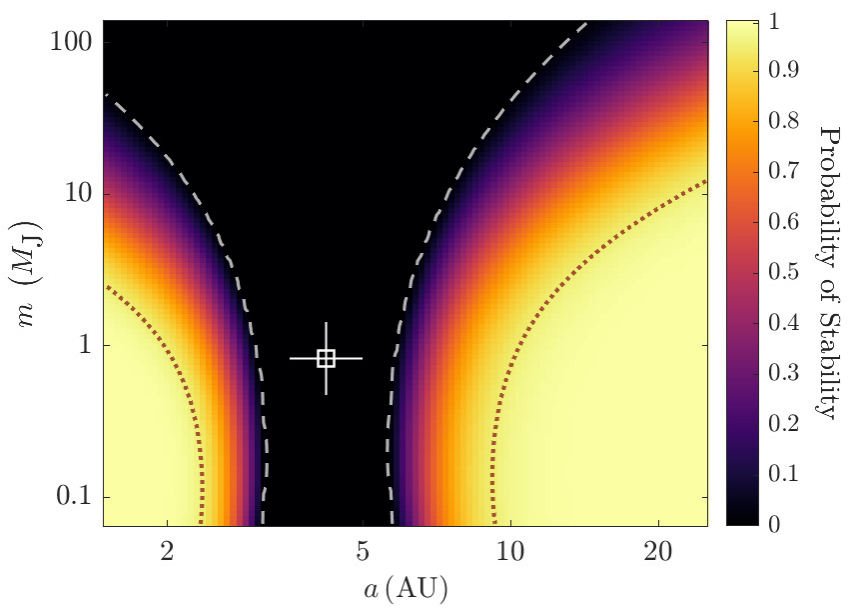

HD 114613 - Petrovich's Criterion

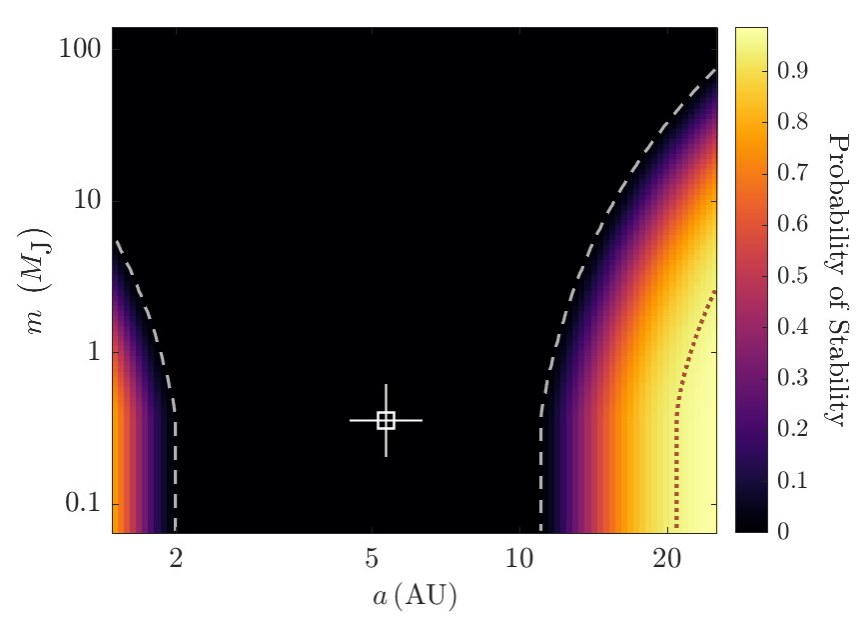

HD 114613 - Giuppone's Mod. Criterion

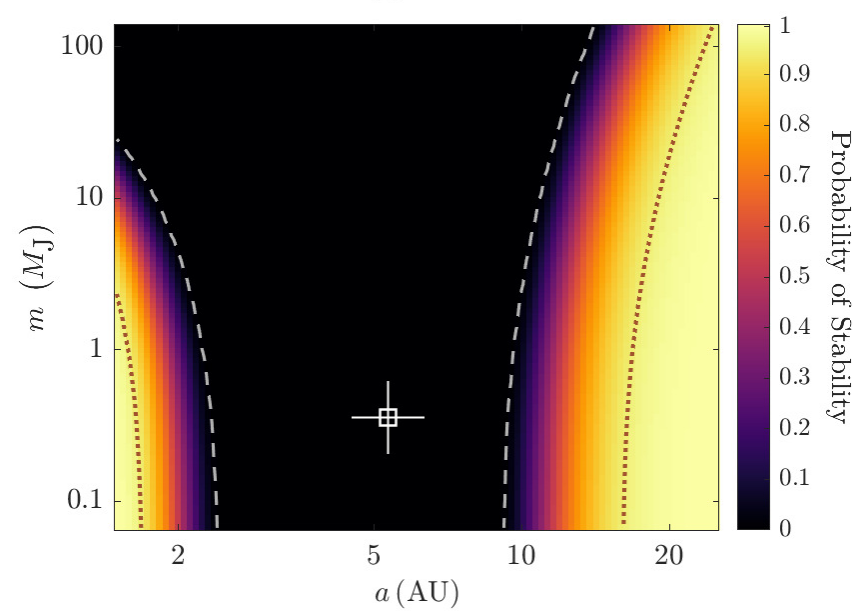

HD 114613 - Hill AMD Criterion

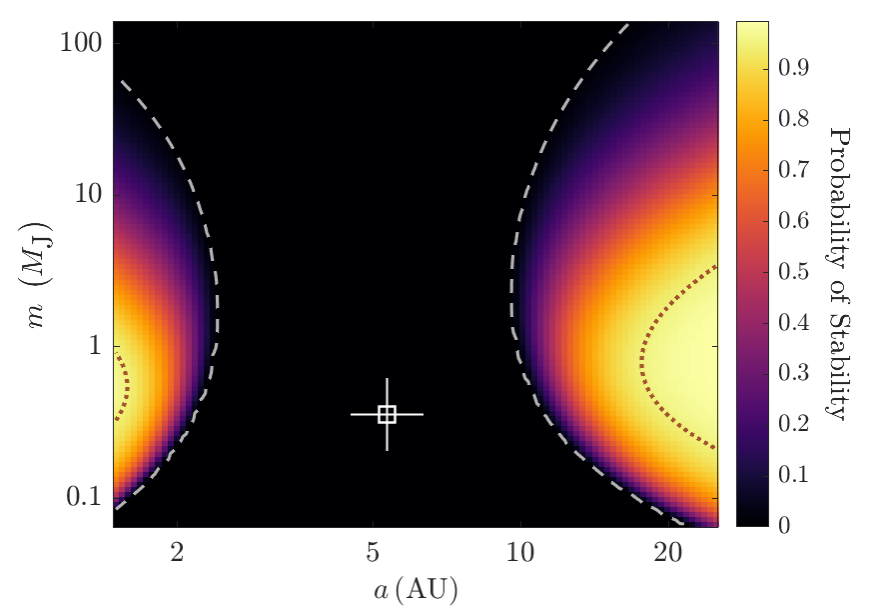

Figure 2. (Modified Figure) Analytic stability maps for the systems HD 154345 (left column) and HD 114613 (right column), using Petrovich's empirical criterion (top row), Giuppone's modified criterion (middle row) and the Hill AMD criterion (bottom row). The white marker indicates the position of the existing planet, the dashed gray line delimits the stable region in the circular case and the dotted brown curve is the 0.95 contour line. 
Table 2. $C$ and $S$ parameters of the FORECASTER modified fit

\begin{tabular}{ccc}
\hline \hline$m\left(M_{\oplus}\right)$ & $C$ & $S$ \\
\hline$m \leq 2.04$ & 0.00346053 & 0.279 \\
$2.04<m \leq 95.16$ & -0.06613329 & 0.50376436 \\
$95.16<m \leq 317.828407$ & 0.48091861 & 0.22725968 \\
$317.828407<m \leq 26635.6863$ & 1.04956612 & 0 \\
$m>26635.6863$ & -2.84926757 & 0.881 \\
\hline
\end{tabular}

our modified fit is in any way more 'correct' than the original FORECASTER model. Rather, as we are focusing on only the larger orbits amenable to direct imaging, we wish to avoid generating planetary mass objects of greater than 1 Jupiter radius, which are expected to be exceedingly rare at the relevant separations. As the original fit also passes quite near the Saturn mass-radius point, we chose to explicitly incorporate Saturn's density in our modification.

While the general procedure and calculations presented in the coming sections rely on the use of a Rayleigh distribution for the eccentricity, we note that the results from Moorhead et al. (2011) were obtained from a sample consisting mostly of intermediate and low-mass planets, and are therefore potentially applicable to only certain regions of the parameter space considered. The range of masses defined by the imageable region directly depends on the system's distance, and therefore, the adequacy of a particular distribution will be essentially determined by the region involved. For instance, in the case of the closest and more interesting targets where lower mass planets are included, the use of the distribution from Moorhead et al. (2011) is certainly convenient. Furthermore, the assumption of a Rayleigh distribution allows for the derivation of a simple and easy to compute expression, which might be more helpful when analyzing large target lists. Alternatively, in the case of the higher-mass regions also explored in our study, the use of, for instance, the Beta distribution proposed by Kipping (2013) may also be a suitable alternative worth considering. To do so, in Figure 3, we reproduce two sample cases assuming that the unknown planet's eccentricity follows a $\operatorname{Beta}(\mathrm{a}, \mathrm{b})$ distribution with parameters $a=0.867$ and $b=3.03$, while the systems and stability criteria remain the same. As we can see, despite certain differences regarding the spatial evolution of the probability of stability, the resulting stability maps bear a considerable resemblance to the figures obtained with the Rayleigh distribution, with only small variations in the overall results yielded by the complete analysis performed in the following sections.

\section{SINGLE-PLANET SYSTEMS PRIORITIZATION}

Having constructed the analytic stability maps, we now address the main purpose of this study by identifying which single-planet systems are more likely to host an additional, imageable planet. Basically, the proposed methodology consists of estimating and comparing the expected number of planets (i.e., occurrence rates) within each system's stable-imageable region defined in the $(a, R)$ space. To do so, together with the stability maps obtained in Section 4, we shall make use of the following additional grids:

1. Depth-of-search grid: Given a particular system, we obtain its imageable region by computing the depth-of-search grids as defined by Garrett et al. (2017). For given values of $a$ and $R$, the corresponding bin represents the conditional probability of detecting a hypothetically existing planet (i.e., completeness; Brown 2005) according to the considered instrument's design and capabilities. In particular, here we set the necessary optical parameters and contrast limits according to the CGI's inner and outer working angles and predicted contrast curve in the $575 \mathrm{~nm}$ imaging band. For planet photometry, we use the model grids from Batalha et al. (2018), which are interpolated to find the phase curves of various planets in reflected light.

2. Occurrence grid: In order to calculate the expected number of planets in a certain region, we build occurrence grids using the SAG 13 parametric fit for G-dwarfs. Similarly to Garrett \& Savransky (2018), we translate the 
HD 154345 - Hill AMD Criterion - Beta

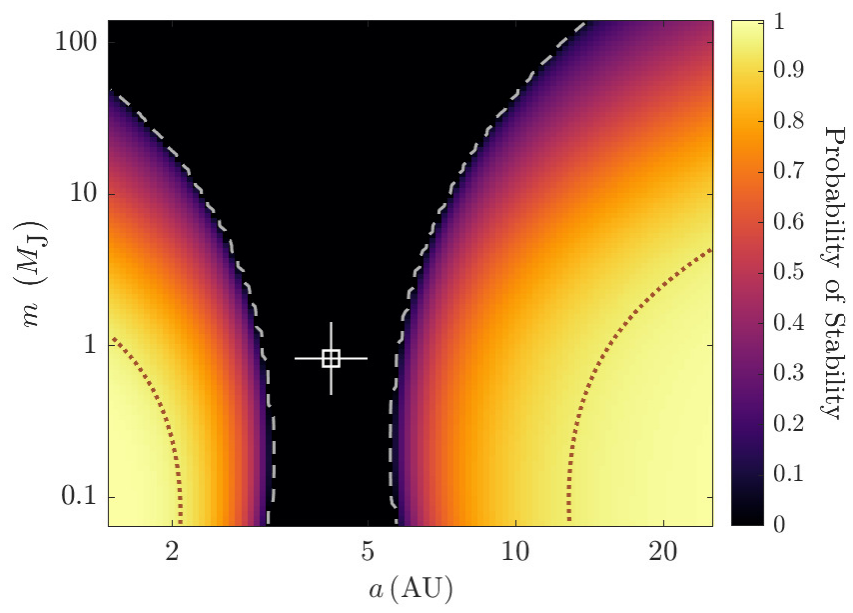

HD 114613 - Petrovich's Criterion - Beta

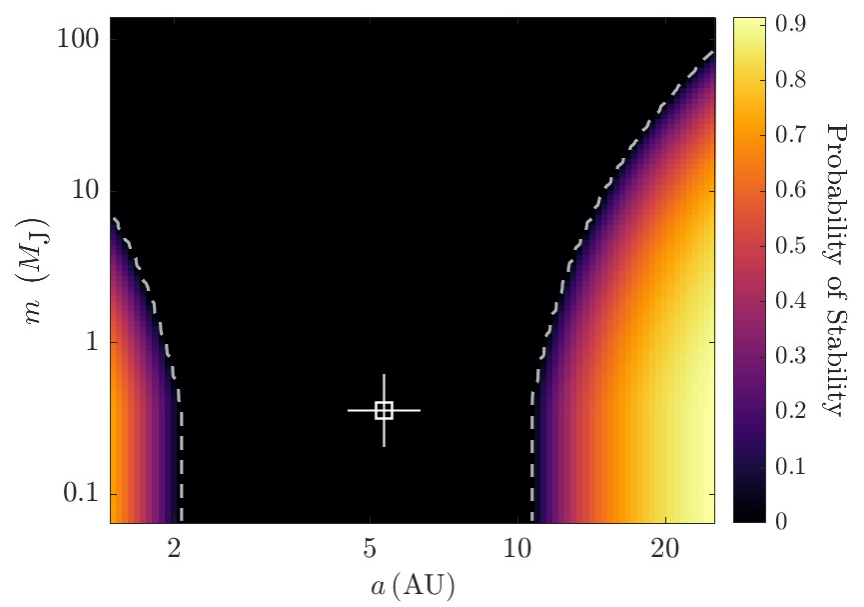

Figure 3. (New Figure) Analytic stability maps assuming the eccentricity follows a beta distribution, for the systems HD 154345 (left) and HD 114613 (right), using the Hill AMD and Petrovich's criterion, respectively. The white marker indicates the position of the existing planet, the dashed gray line delimits the stable region in the circular case and the dotted brown curve is the 0.95 contour line.

Table 3. (New Table)

SAG13 parametric fit parameters.

\begin{tabular}{cccc}
\hline \hline$R\left(R_{\oplus}\right)$ & $\Gamma$ & $\alpha$ & $\beta$ \\
\hline$R<3.4$ & 0.38 & -0.19 & 0.26 \\
$R \geq 3.4$ & 0.73 & -1.18 & 0.59 \\
\hline
\end{tabular}

original period-radius broken power law into the $(a, R)$ space and we add an exponential decay term starting at $a_{k}=10 \mathrm{AU}$. The resulting parametric model for the occurrence rate $\eta$ is then given by

$$
\frac{\partial^{2} \eta}{\partial R \partial a}=\Gamma_{i} R^{\alpha_{i}-1}\left(2 \pi \sqrt{\frac{a^{3}}{\mu}}\right)^{\beta_{i}-1}\left(3 \pi \sqrt{\frac{a}{\mu}}\right) \exp \left(-\left(\frac{a}{a_{k}}\right)^{3}\right),
$$

where the values of the original SAG13 fit parameters $\Gamma, \alpha$ and $\beta$ can be found in Table 3 .

Given that the size of the imageable region strongly depends on the distance from the observer to the target star, all three grids are ranged according to each system's detection boundaries $\left(a_{\min }, a_{\max }\right.$ and $\left.R_{\min }\right)$. This is intended to increase the accuracy of the results by only calculating stability around the imageable zone. In general, the maximum planetary radius is set to $R_{\max }=17 R_{\oplus}$, since we only wish to consider bodies near the planetary mass regime. For the semi-major axis, the inner limit will be essentially determined by the minimum projected separation

$$
a_{\min }=s_{\min }=I W A \cdot d
$$

where $I W A$ is the telescope's inner working angle and $d$ is the distance to the system. To find the maximum value of $a$, we consider the expression for the ratio of fluxes between the planet and the star (Brown 2005)

$$
F_{R}=p \Phi(\beta)\left(\frac{R}{r}\right)^{2}
$$

where $p$ and $\beta$ are the planet's albedo and phase angle, respectively, $\Phi$ is the phase function and $r$ is the distance between the planet and the star. For a particular value of $R$, the upper limit of the imageable region is characterized 
by the maximum $a$ such that the planet meets the instrument's obscurational and photometric constraints, determined by $s_{\min }$ and the expected mininum contrast $c_{\min }$. These values can be related to the upper boundary of the nonzero region of the completeness joint probability density function (Garrett \& Savransky 2016) given by one of the solutions of

$$
F(a \mid R)=c_{\min }-p \Phi\left[\sin ^{-1}\left(\frac{s_{\min }}{a}\right)\right]\left(\frac{R}{a}\right)^{2}=0,
$$

where $r$ has been replaced by $a$, since the depth-of-search grids are defined assuming that $e=0$ (Garrett et al. 2017). Since the width of the imageable region increases with $R$, the maximum semi-major axis $a_{\max }$ is consequently given by the upper bounding solution of the equation $F\left(a \mid R_{\max }\right)=0$, where again $R_{\max }$ is the largest planetary radius considered. Having determined $a_{\min }$ and $a_{\max }$, we can finally obtain the minimum radius by isolating $R$ in Equation (36) and calculating

$$
R_{\min }=\min _{a \in\left(a_{\min }, a_{\max }\right)}\left\{\sqrt{\frac{a^{2} c_{\min }}{p \Phi\left(\sin ^{-1}\left(s_{\min } / a\right)\right)}}\right\} .
$$

For each system, the product of the stability and depth-of-search grids yields the intersection between the stable and imageable regions, where the value of each bin gives the probability of detecting a stable planet of radius $R$ and semimajor axis $a$. For instance, in Figure 4 we represent the resultant grids for the systems HD 154345 and HD 114613, using the Hill AMD and Petrovich's criteria respectively. In order to properly identify the limits of the nonzero regions, the bins with null probability are not coloured.

As expected, in both cases the size of the imageable region is clearly defined by the solutions to the equations presented above. Such limits, together with the estimated detection probability of each bin, are only a function of the distance to the system and the instrument's capabilities. In consequence, the resemblance between both depth-ofsearch grids can be directly related to the similar target distance (see Table 1), while also evidencing that the results ignore integration time constraints since there is no dependence on the magnitude of the star. Regarding the $(a, R)$ stability maps, we first note a clear contrast between the extension of the unstable regions of both systems, essentially as a result of the difference in the orbital eccentricities of the existing planets and the stability criteria employed. Moreover, the use of the modified FORECASTER best-fit described in Section 4 results in a discontinuous increase in planetary mass occurring at $1 R_{\mathrm{J}}\left(\approx 11.2 R_{\oplus}\right)$, causing the steep growth in the unstable region observed at that point. In general, the stable-imageable grid demonstrates how the depth-of-search is highly perturbed by the region where planets cannot exist due to instabilities, confirming that the presence of the known planet should be considered when optimizing the target selection. The sum over the intersection bins, normalized by the number of bins and multiplied by the grid area, yields what we refer to as the dynamically stable depth-of-search. This value has no dependence on the assumed planet population and only accounts for the considered instrument's performance and stability criterion. Finally, the convolution of the intersection region with the occurrence grid returns the desired estimate of the expected number of stable and imageable planets in the system (dynamically stable completeness), yielding a metric that can be directly used for imaging prioritization.

We apply this procedure to all 213 currently known single-planet systems within a distance of $50 \mathrm{pc}$, creating a ranking based on Petrovich's stability criterion and complemented by the results obtained with Giuppone's and the Hill AMD criteria (Appendix A). Naturally, the results show a clear dependence on the system's distance $d$, generally making the closest targets the most valuable. Nonetheless, we also note how some of the nearest stars present a lower dynamically stable completeness in comparison to farther targets with smaller imageable regions. This is clearly observed in Figure 6, where the stable completeness for every target analyzed and stability criteria used, as a function of the system's distance, is represented. As a result of the dependence of the imageable region on the distance to the system, the expected number of stable and imageable planets decreases along the horizontal axis. Those targets where the presence of the known planet has a greater effect present a lower stable completeness, consequently falling below the main tendency defined by the upper line. Comparing the results given by each stability criterion, we observe how Giuppone's criterion usually returns the highest completeness values, while Petrovich's and the Hill AMD criteria, in accordance with the behaviors observed in Section 2.3, tend to give more conservative results. Although we are focusing on the search of unknown companions, the systems where the already existing exoplanet falls inside the imageable region (such as HD 154345 or HD 114613) are still interesting targets, given that the majority of known exoplanets have been discovered with indirect detection techniques and still need to be directly imaged in order to obtain spectral information, which will allow for modeling currently unknown properties such as their 
HD 154345 - Depth-of-Search Grid

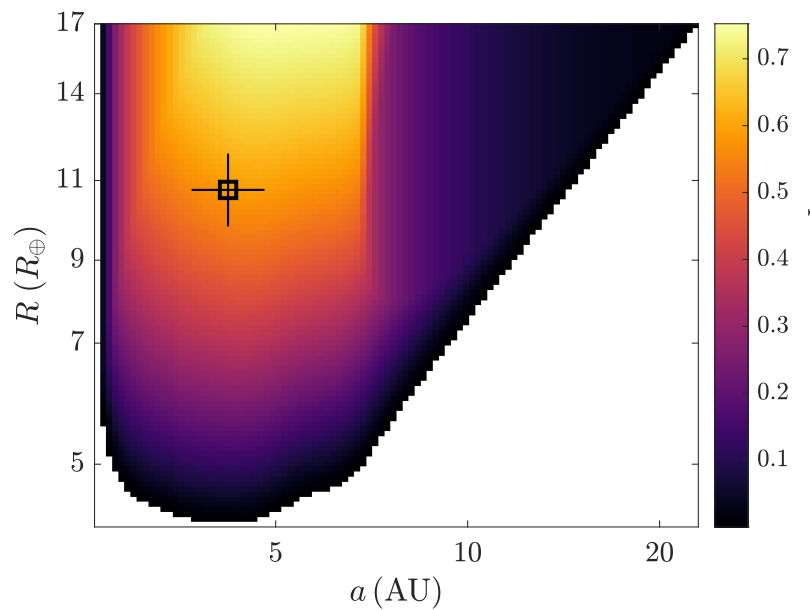

HD 154345 - Stability Grid - Hill AMD Crit.

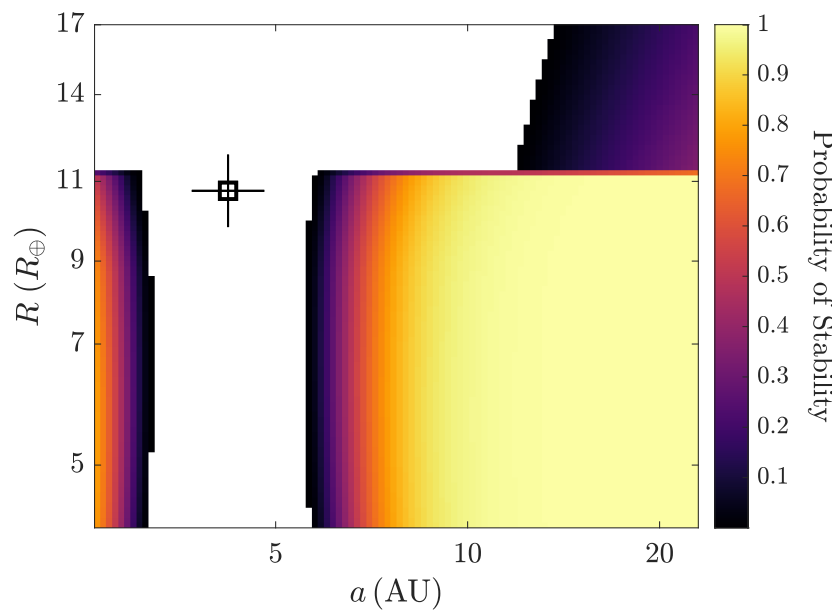

HD 154345 - Intersection Grid - Hill AMD Crit.

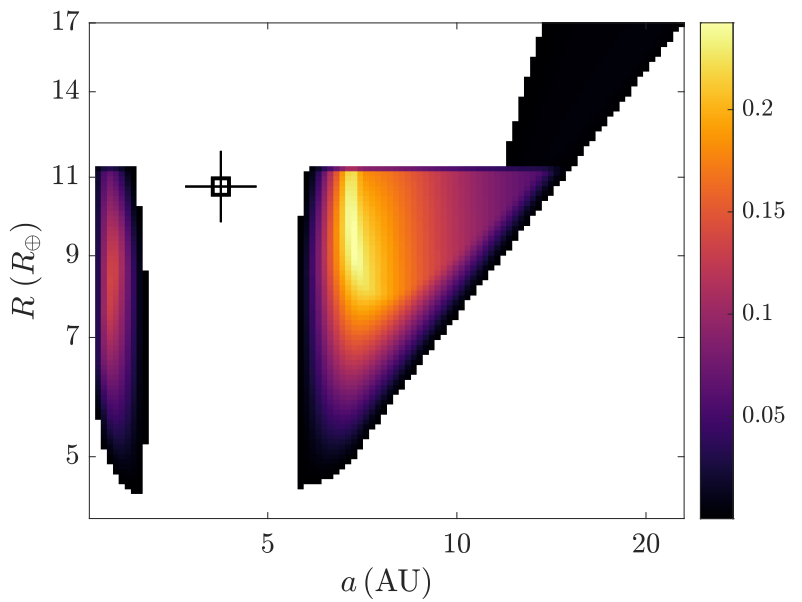

HD 114613 - Depth-of-Search Grid

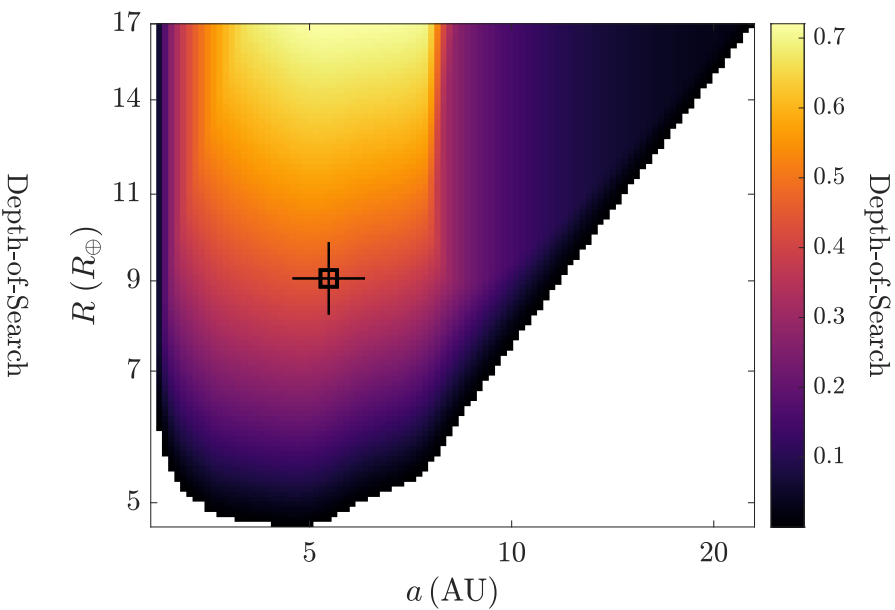

HD 114613 - Stability Grid - Petrovich's Crit.

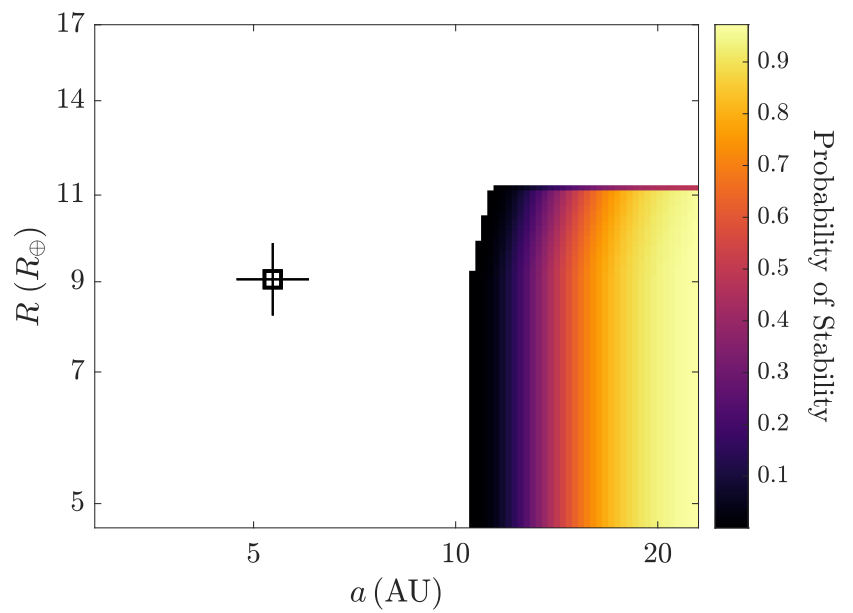

HD 114613 - Intersection Grid - Petrovich's Crit.

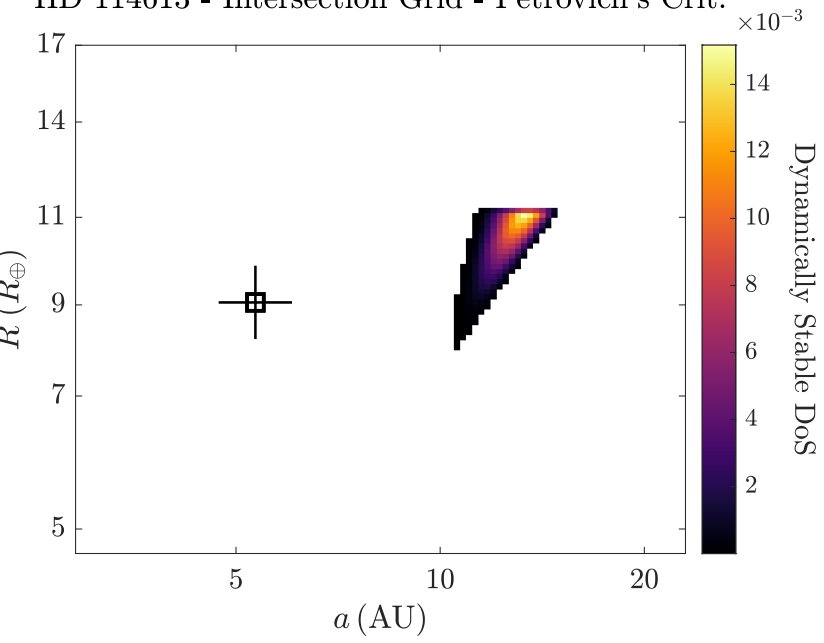

Figure 4. Depth-of-Search (top), Stability (middle) and Intersection (bottom) grids for the system HD 154345 using the Hill AMD criterion (left), and the system HD 114613 using Petrovich's criterion (right). The black marker indicates the position of the existing planet. 
GJ 649 - Depth-of-Search Grid

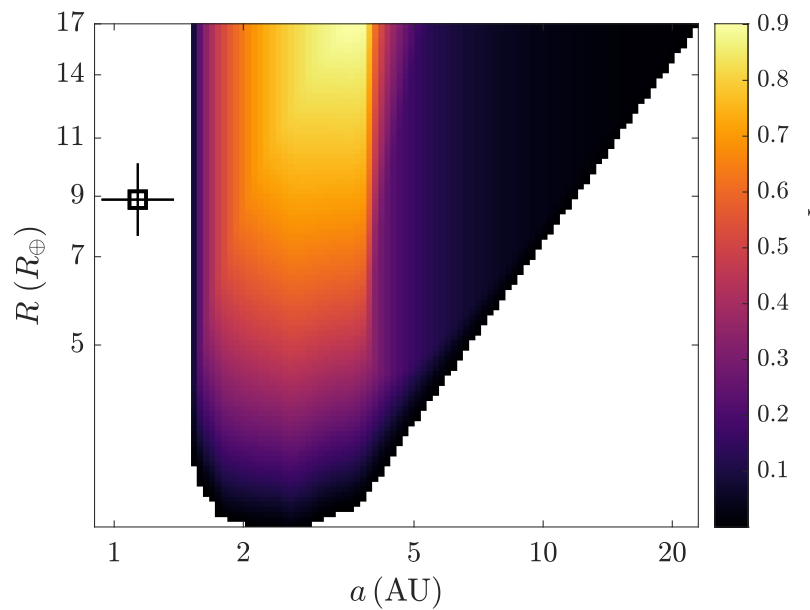

GJ 649 - Stability Grid - Giuppone's Mod. Crit.

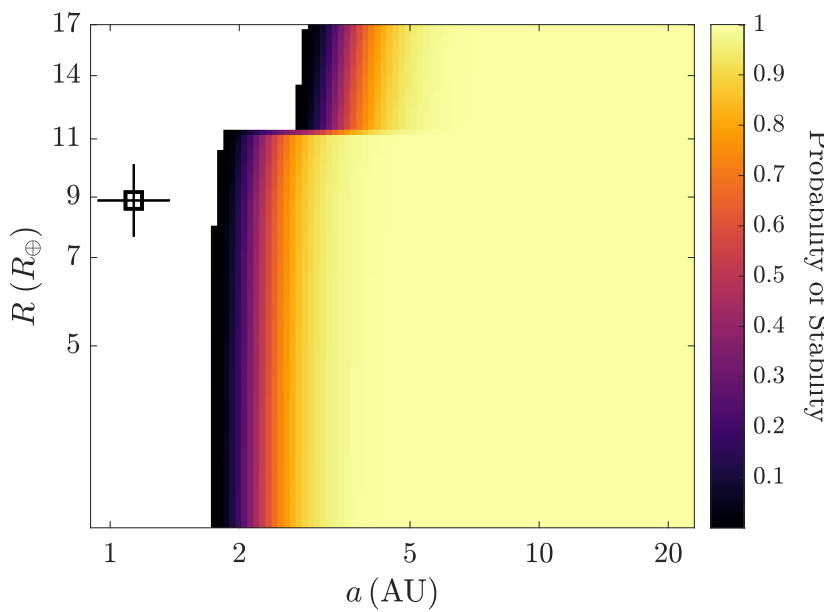

GJ 649 - Intersection Grid - Giuppone's Mod. Crit.

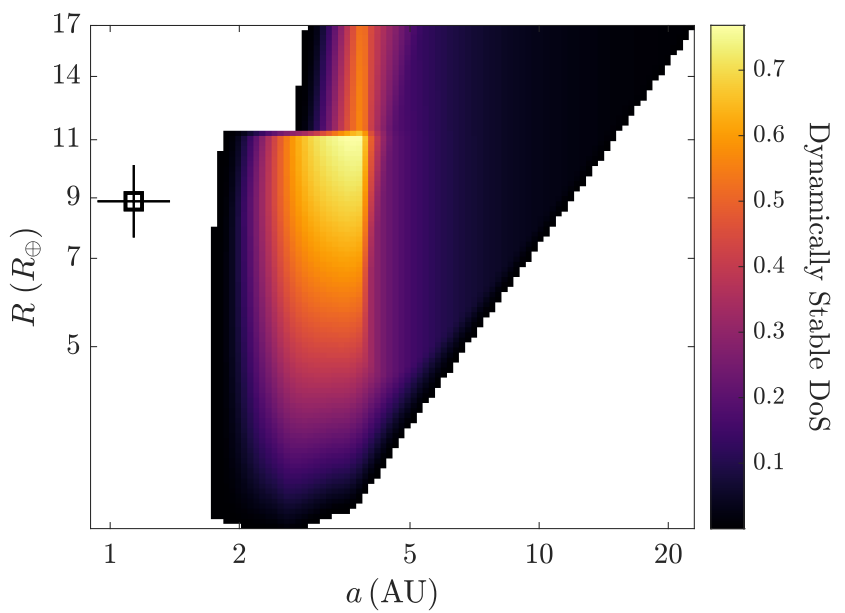

HD 221420 - Depth-of-Search Grid

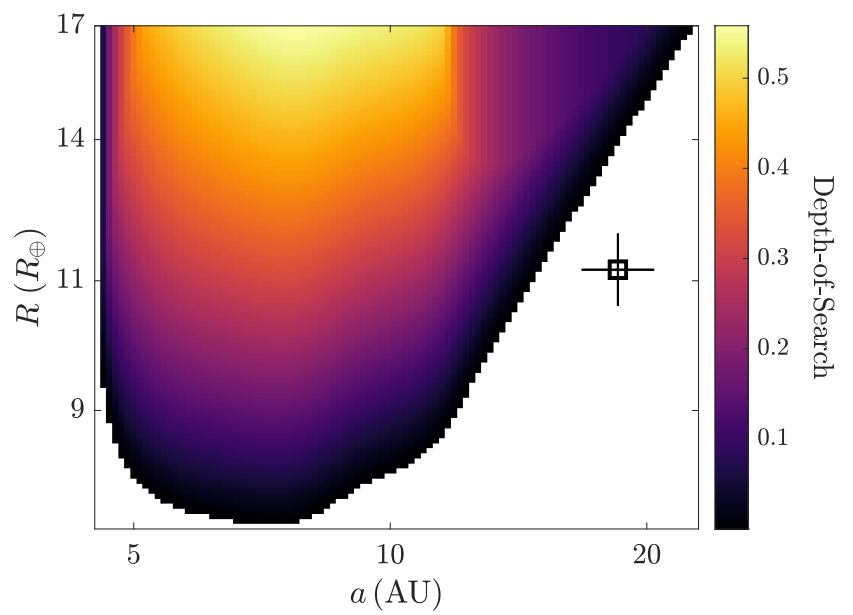

HD 221420 - Stability Grid - Petrovich's Crit.

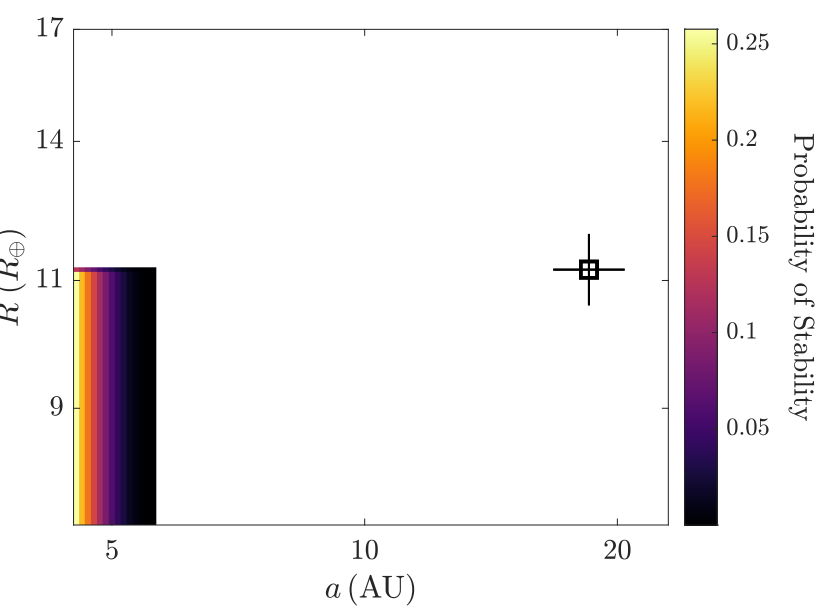

HD 221420 - Intersection Grid - Petrovich's Crit.

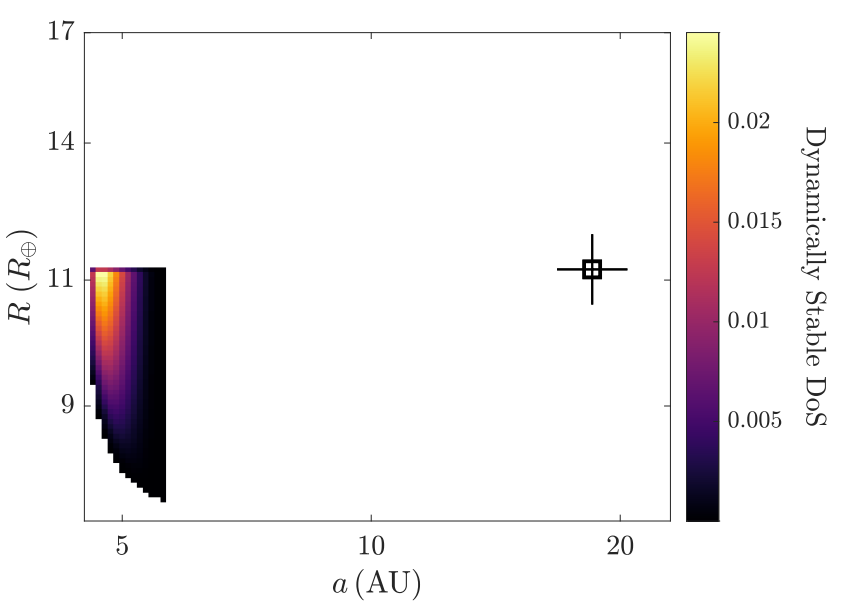

Figure 5. Depth-of-Search (top), Stability (middle) and Intersection (bottom) grids for the system GJ 649 using Giuppone's modified criterion (left), and the system HD 221420 using Petrovich's criterion (right). The black marker indicates the position of the existing planet. 


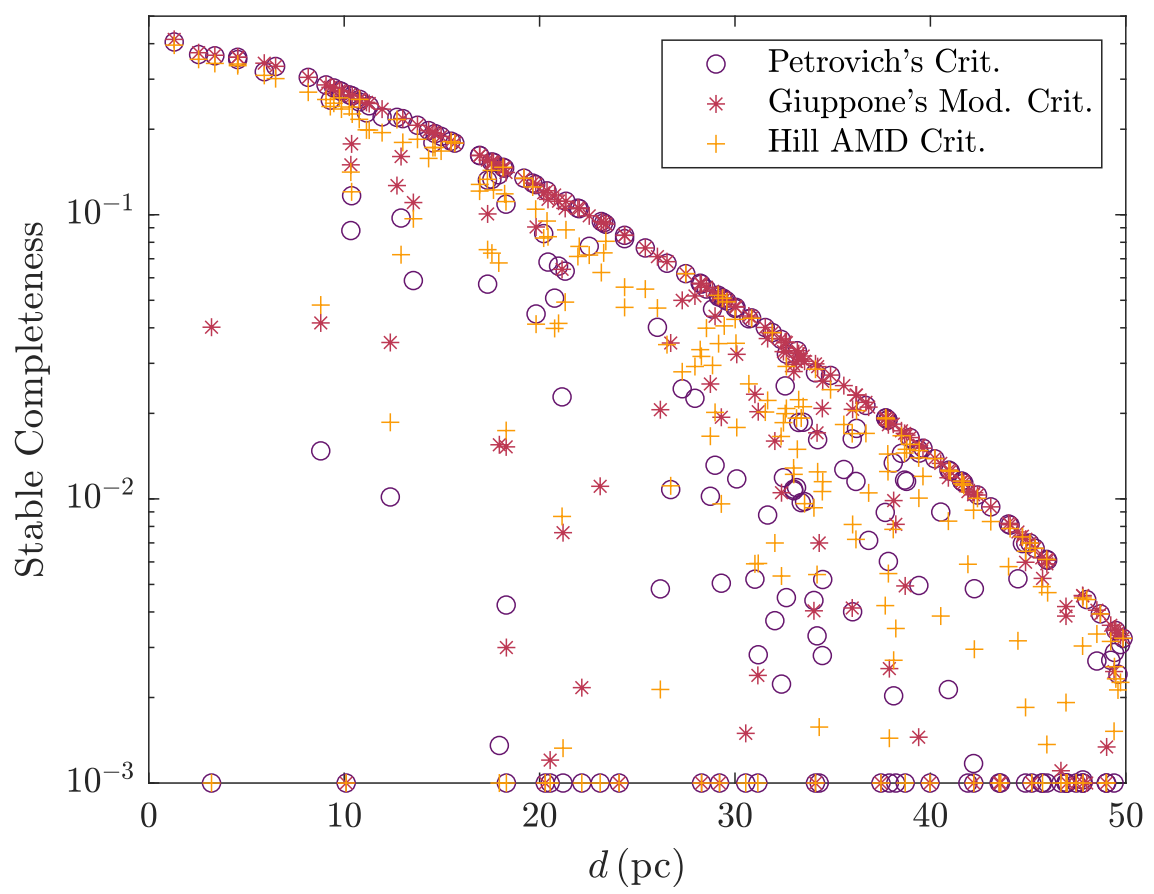

Figure 6. Stable completeness as a function of the system's distance. For all targets, the estimated number of imageable and stable planets according to each stability criterion is plotted. Completeness values below $10^{-3}$ are plotted over the horizontal axis.

atmospheric structure or chemical composition. In Figure 5 we present the resultant grids for the systems GJ 649 and HD 221420, where the existing planet is located outside the lower and upper limits of the imageable region, respectively. These plots clearly demonstrate how the presence of the known planets, which are themselves unobservable with the assumed instrument, still impacts the likelihood of observing other potential planets in these systems.

Furthermore, in order to complete our study, we should also take into account the radial velocity data obtained from past Doppler surveys. In this sense, the exploration and subsequent non-detection of additional planets in some of the targets considered may help us rule out entire regions of our parameter space where, if a planet existed, it would have certainly been detected by previous RV observations. To do so, we directly apply the results from Howard \& Fulton (2016), where they use measurements at both Lick and Keck Observatories taken by the California Planet Survey in order to evaluate the probability of recovering an additionally injected synthetic RV signal simulating the presence of a planet of mass $m \sin I$ and semi-major axis $a$. In particular, for each target considered, they introduce 5000 synthetic planets uniformly distributed and apply an iterative planet search algorithm to attempt to detect the injected signals, ultimately obtaining a spatial distribution of the corresponding recovery rates. They apply this procedure to a target list containing 76 nearby stars, 6 of which were single-planet systems also present in our star sample. While repeating the recovery tests for the remaining 207 stars included in our target list is beyond the scope of this work, we can make use of the available calculations by combining our dynamically stable completeness maps with the results presented by Howard \& Fulton (2016). Regarding the latter, due to the differences between the range of values employed in each study, we need to carefully translate the recovery rate distribution from Howard \& Fulton (2016) into our parameter space and grid values. To do so, we build our adapted contour plots by linearly interpolating the recovery rates corresponding to the grid points falling inside their original range of values. On the other hand, for the higher planetary masses considered in the present study, we follow the vertical trend observed in the original contour plots and perform a uniform extrapolation of the recovery rates associated with the upper mass limits evaluated in Howard \& Fulton (2016). This entire procedure is demonstrated in the left-hand plot of Figure 7, where the distribution of recovery rates for the system HD 3651, adapted to our parameter space and range of values, is represented. In particular, 
Table 4. (New Table) Doppler corrected dynamically stable completeness.

\begin{tabular}{|c|c|c|c|c|c|c|c|c|c|c|}
\hline \multicolumn{2}{|c|}{ Target } & \multicolumn{3}{|c|}{ Known Planet } & \multicolumn{3}{|c|}{ Stable Completeness } & \multicolumn{3}{|c|}{ Doppler Corrected Completeness } \\
\hline Name & $\begin{array}{c}\text { Distance } \\
(\mathrm{pc})\end{array}$ & $\begin{array}{c}a_{k} \\
(\mathrm{AU})\end{array}$ & $e_{k}$ & $\begin{array}{c}m_{k} \sin I \\
\left(M_{\mathrm{J}}\right)\end{array}$ & $\begin{array}{r}\text { Petrovich } \\
\qquad(\mathrm{S}\end{array}$ & $\begin{array}{l}\text { Giuppone } \\
\text { ability crite }\end{array}$ & $\begin{array}{l}\text { Hill AMD } \\
\text { ia) }\end{array}$ & $\begin{array}{r}\text { Petrovich } \\
\text { (S }\end{array}$ & $\begin{array}{l}\text { Giuppone } \\
\text { ability crite }\end{array}$ & Hill AMD \\
\hline HD 3651 & 11.14 & 0.295 & 0.645 & 0.228 & 0.22829 & 0.24871 & 0.19898 & 0.13682 & 0.13693 & 0.11859 \\
\hline $51 \mathrm{Peg}$ & 15.47 & 0.053 & 0.013 & 0.472 & 0.18147 & 0.18147 & 0.18074 & 0.10399 & 0.10399 & 0.10399 \\
\hline tau Boo & 15.66 & 0.049 & 0.011 & 4.32 & 0.17870 & 0.17870 & 0.17870 & 0.12827 & 0.12827 & 0.12827 \\
\hline $70 \mathrm{Vir}$ & 17.91 & 0.481 & 0.399 & 7.416 & 0.13822 & 0.14952 & 0.06767 & 0.08031 & 0.08291 & 0.01935 \\
\hline HD 19994 & 22.54 & 1.305 & 0.063 & 1.37 & 0.07743 & 0.09913 & 0.07212 & 0.05941 & 0.06159 & 0.05799 \\
\hline eps Eri & 3.21 & 3.39 & 0.702 & 1.55 & 0.00046 & 0.04020 & 0.00016 & 0.00043 & 0.03795 & 0.00000 \\
\hline
\end{tabular}

the gray dashed line indicates the mass above which the results were uniformly extrapolated, while the lower values were linearly interpolated. Similarly, the equivalent $(a, R)$ maps can be constructed (right-hand plot of Figure 7), where now the colour map represents the fraction of non-recovered planets, which we will refer to as Doppler insensitivity. In this sense, in the regions where the Doppler insensitivity decreases (i.e., the fraction recovered is high), an additional planet would have already been detected by past RV surveys and therefore the corresponding probability of finding a new companion is lower. Furthermore, by combining the resulting Doppler insensitivity maps with our stable-imageable regions in the $(a, R)$ space, we can compute the Doppler corrected, dynamically stable completeness associated with the six single-planet systems considered, the values of which can be found in Table 4 . As we can observe, the consideration of the radial velocity data reduces significantly the target's total completeness, consequently suggesting that, in future studies, a detailed Doppler analysis for the most interesting targets would be needed in order to improve and complement our prioritization.

While the target ranking presented in Appendix A is certainly incomplete without the addition of Doppler insensitivity for all of the tabulated systems, the true utility of this table is in its index of the underlying depth of search and stability grids. These represent more than half of the effort involved in the final determination of the utility of direct imaging of these targets in search for unknown companions. These grids can be combined with updated Doppler completeness values as these become available, in order to produce up to the minute target rankings at the time a mission is launched.

\section{CONCLUSIONS}

Running numerical simulations up to $10^{9}$ years, we have analyzed and compared various stability criteria for twoplanet systems with arbitrary eccentricities, showing that the criterion from Petrovich (2015) is generally the most conservative and useful for our purposes, although the stability limits defined by Giuppone et al. (2013) and Petit et al. (2018) also perform reasonably well. The code used to perform the numerical simulations is publicly available at https://github.com/CarlosGascon/NumSim. For any criterion expressed as a boundary of the outer pericenter to inner apocenter ratio $(\rho)$ or the angular momentum deficit $(C)$, we have derived expressions for the conditional probability of having a stable companion given fixed values of $a$ and $m$. This formulation has been directly used for the computation of analytic stability maps, allowing us to rapidly characterize the stable region of a system in the $(a, R)$ space. By intersecting with the depth-of-search grids defined by Garrett et al. (2017), we have obtained the corresponding stable-imageable region, yielding the definition of the total dynamically stable depth-of-search, with no dependence on the assumed planet population. In particular, we have presented two cases where the detectable region is clearly perturbed by the stability boundaries, showing the importance of accounting for the effects of the existing planet in such systems. Furthermore, we added two examples of systems where the existing planet falls outside the imageable region but its gravitational effect is still noticeable. Finally, the convolution with the selected occurrence grid has allowed us to estimate the expected number of stable and imageable planets in a system. Applying this procedure to all the currently known single-planet systems and several stability criteria, we have built a ranked target 

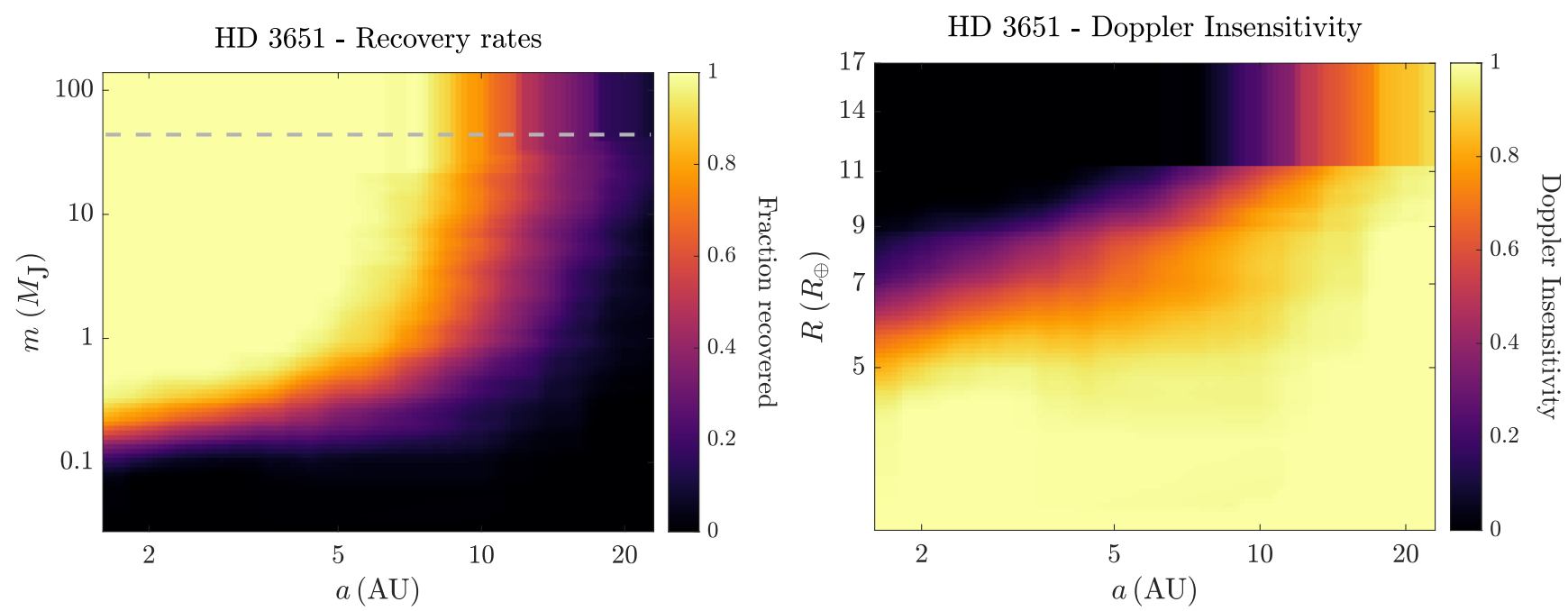

Figure 7. (New Figure) Recovery rates (left) and Doppler insensitivity map (i.e., fraction of non-recovered planets) in the $(a, R)$ space (right) for the system HD 3651. Colour maps were built by translating the results from Howard \& Fulton (2016) into our parameter space. In particular, the gray dashed line in the left figure indicates the mass above which the original recovery rates had to be extrapolated following the observed vertical trend.

list based on the CGI's capabilities and the SAG13 parametric fit. Additionally, we have completed our study by considering the radial velocity data from past Doppler surveys and evaluating how the non-detection of additional planets in some of the targets can help us rule out entire regions of our parameter space. Making use of the results from Howard \& Fulton (2016), we have observed that the consideration of the radial velocity data reduces significantly the target's total completeness, consequently suggesting that, in future studies, a detailed Doppler analysis for the most interesting targets would be needed in order to improve and complement our prioritization. The code used for both the construction of analytic stability maps and the systems prioritization is publicly available at https://github.com/CarlosGascon/StableDoS. The generated depth of search and stability grids are all available via Cornell eCommons at [url to be filled in at time of manuscript acceptance]. Although a numerical analysis could lead to more accurate results, the proposed methodology is a powerful tool, not only for rapidly identifying which targets have a higher probability of hosting an additional planet, but also for discarding those systems where no unknown companions can be detected.

This research made use of the NASA Exoplanet Archive, which is operated by the California Institute of Technology, under contract with the National Aeronautics and Space Administration under the Exoplanet Exploration Program. In addition, this research employed the Imaging Mission Database, which is operated by the Space Imaging and Optical Systems Lab (SIOSlab) at Cornell University. C. G. would also like to thank the Interdisciplinary Higher Education Center (CFIS) from the Polytechnic University of Catalonia (UPC), as well as the CELLEX Foundation, for offering him the opportunity of developing this work at Cornell University.

\section{REFERENCES}

Agnew, M. T., Maddison, S. T., \& Horner, J. 2018, Monthly

Notices of the Royal Astronomical Society, 481, 4680

Batalha, N. E., Smith, A. J., Lewis, N. K., et al. 2018, The

Astronomical Journal, 156, 158

Brown, R. A. 2005, The Astrophysical Journal, 624, 1010

Chen, J., \& Kipping, D. 2016, The Astrophysical Journal, 834,17
Deck, K. M., Payne, M., \& Holman, M. J. 2013, The Astrophysical Journal, 774, 129

Duncan, M., Quinn, T., \& Tremaine, S. 1989, Icarus, 82, 402

Garrett, D., \& Savransky, D. 2016, The Astrophysical Journal, 828, 20

Garrett, D., \& Savransky, D. 2018, in American Astronomical Society, AAS Meeting, Vol. 231 
Garrett, D., Savransky, D., \& Macintosh, B. 2017, The Astronomical Journal, 154, 47

Giuppone, C., Morais, M., \& Correia, A. 2013, Monthly Notices of the Royal Astronomical Society, 436, 3547

Gladman, B. 1993, Icarus, 106, 247

Hadden, S., \& Lithwick, Y. 2018, The Astronomical Journal, 156, 95

Howard, A. W., \& Fulton, B. J. 2016, Publications of the Astronomical Society of the Pacific, 128, 114401

Kipping, D. M. 2013, Monthly Notices of the Royal Astronomical Society: Letters, 434, L51

Konopacky, Q. M., Barman, T. S., Macintosh, B. A., \& Marois, C. 2013, Science, 339, 1398
Laskar, J. 2000, Physical Review Letters, 84, 3240

Laskar, J., \& Petit, A. 2017, Astronomy \& Astrophysics, 605, A72

Marchal, C., \& Bozis, G. 1982, Celestial Mechanics, 26, 311

Moorhead, A. V., Ford, E. B., Morehead, R. C., et al. 2011, The Astrophysical Journal Supplement Series, 197, 1

Petit, A. C., Laskar, J., \& Boué, G. 2017, Astronomy \& Astrophysics, 607, A35

Petit, A. C., Laskar, J., \& Boué, G. 2018, A\&A, 617, A93

Petrovich, C. 2015, The Astrophysical Journal, 808, 120

Rein, H., \& Liu, S.-F. 2012, Astronomy \& Astrophysics, 537, A128

Wisdom, J. 1980, The Astronomical Journal, 85, 1122 


\section{APPENDIX}

\section{A. SINGLE-PLANET SYSTEMS RANKING}

Table 5. Dynamically stable depth-of-search and completeness values (We would like this table to appear in machine readable format).

\begin{tabular}{|c|c|c|c|c|c|c|c|c|c|c|}
\hline \multicolumn{2}{|c|}{ Target } & \multicolumn{3}{|c|}{ Known Planet } & \multicolumn{2}{|c|}{ Petrovich's Crit. } & \multicolumn{2}{|c|}{ Giuppone's Crit. } & \multicolumn{2}{|c|}{ Hill AMD Crit. } \\
\hline Name & $\begin{array}{c}\text { Distance } \\
\text { (pc) }\end{array}$ & $\begin{array}{c}a_{k} \\
(\mathrm{AU})\end{array}$ & $e_{k}$ & $\begin{array}{c}m_{k} \sin I \\
\left(M_{\mathrm{J}}\right)\end{array}$ & $\begin{array}{l}\text { DoS } \\
\text { (Dyna }\end{array}$ & $\begin{array}{l}\text { Completeness } \\
\text { nically Stable) }\end{array}$ & $\begin{array}{l}\text { DoS } \\
\text { (Dyne }\end{array}$ & $\begin{array}{l}\text { Completeness } \\
\text { nically Stable) }\end{array}$ & $\begin{array}{l}\text { DoS } \\
\text { (Dyna }\end{array}$ & $\begin{array}{l}\text { Completeness } \\
\text { mically Stable) }\end{array}$ \\
\hline Proxima Cen & 1.30 & 0.049 & 0.350 & 0.004 & 44.25 & 0.40553 & 52.07 & 0.41418 & 39.39 & 0.39538 \\
\hline GJ 411 & 2.55 & 0.079 & 0.220 & 0.009 & 52.76 & 0.36637 & 57.83 & 0.37178 & 45.12 & 0.35245 \\
\hline Ross 128 & 3.38 & 0.050 & 0.116 & 0.004 & 56.37 & 0.36255 & 57.89 & 0.36422 & 44.41 & 0.34088 \\
\hline GJ 674 & 4.55 & 0.039 & 0.200 & 0.035 & 59.15 & 0.35824 & 59.22 & 0.35833 & 48.66 & 0.33925 \\
\hline GJ 687 & 4.55 & 0.170 & 0.040 & 0.060 & 54.40 & 0.35174 & 59.20 & 0.35829 & 46.52 & 0.33474 \\
\hline GJ 625 & 6.49 & 0.078 & 0.130 & 0.009 & 61.58 & 0.33251 & 61.90 & 0.33296 & 46.38 & 0.30137 \\
\hline HD 180617 & 5.91 & 0.336 & 0.160 & 0.038 & 47.40 & 0.31868 & 59.10 & 0.34149 & 44.15 & 0.30944 \\
\hline Gl 686 & 8.16 & 0.092 & 0.077 & 0.021 & 62.30 & 0.30439 & 62.40 & 0.30452 & 45.94 & 0.27006 \\
\hline GJ 433 & 9.07 & 0.060 & 0.080 & 0.020 & 61.39 & 0.28633 & 61.40 & 0.28633 & 46.17 & 0.25446 \\
\hline HD 285968 & 9.47 & 0.066 & 0.000 & 0.026 & 60.72 & 0.27940 & 60.73 & 0.27941 & 45.46 & 0.24692 \\
\hline GJ 436 & 9.76 & 0.029 & 0.138 & $0.070^{\mathrm{b}}$ & 61.41 & 0.27324 & 61.41 & 0.27324 & 53.51 & 0.25779 \\
\hline Gl 49 & 9.86 & 0.091 & 0.363 & 0.018 & 60.91 & 0.27147 & 61.07 & 0.27171 & 44.00 & 0.23557 \\
\hline GJ 1265 & 10.26 & 0.026 & 0.040 & 0.023 & 61.33 & 0.26413 & 61.33 & 0.26413 & 49.20 & 0.23939 \\
\hline GJ 536 & 10.41 & 0.067 & 0.080 & 0.017 & 61.58 & 0.26188 & 61.59 & 0.26189 & 44.52 & 0.22630 \\
\hline GJ 86 & 10.79 & 0.110 & 0.040 & 4.420 & 60.60 & 0.25471 & 60.61 & 0.25473 & 60.59 & 0.25467 \\
\hline HD 102365 & 9.29 & 0.460 & 0.340 & 0.050 & 46.70 & 0.25312 & 60.47 & 0.28076 & 42.20 & 0.24039 \\
\hline HD 147379 & 10.77 & 0.323 & 0.070 & 0.090 & 57.24 & 0.24950 & 60.68 & 0.25505 & 42.53 & 0.21670 \\
\hline HD 85512 & 11.28 & 0.260 & 0.110 & 0.010 & 56.86 & 0.24182 & 59.67 & 0.24641 & 37.91 & 0.19858 \\
\hline HD 3651 & 11.14 & 0.295 & 0.645 & 0.228 & 49.05 & 0.22829 & 60.18 & 0.24871 & 42.89 & 0.19898 \\
\hline $\begin{array}{l}\text { GJ } 96 \\
\text { VHS }\end{array}$ & 11.94 & 0.291 & 0.440 & 0.062 & 51.30 & 0.22102 & 59.49 & 0.23535 & 40.27 & 0.19457 \\
\hline $\begin{array}{l}\text { J125601.92- } \\
125723.9\end{array}$ & 12.70 & 102.0 & $0.000^{\mathrm{a}}$ & 11.200 & 57.47 & 0.22018 & 26.42 & 0.12668 & 55.94 & 0.21635 \\
\hline HD 211970 & 13.00 & 0.143 & 0.150 & 0.041 & 58.40 & 0.21789 & 58.48 & 0.21803 & 40.13 & 0.17971 \\
\hline GJ 3779 & 13.75 & 0.026 & 0.070 & 0.025 & 57.74 & 0.20652 & 57.74 & 0.20652 & 46.62 & 0.18434 \\
\hline GJ 685 & 14.32 & 0.134 & 0.000 & 0.028 & 56.90 & 0.19775 & 56.92 & 0.19778 & 37.48 & 0.15788 \\
\hline GJ 1214 & 14.65 & 0.014 & $0.000^{\mathrm{a}}$ & $0.020^{\mathrm{b}}$ & 56.25 & 0.19296 & 56.25 & 0.19296 & 45.87 & 0.17243 \\
\hline Gl 378 & 14.96 & 0.039 & 0.109 & 0.041 & 56.53 & 0.18869 & 56.53 & 0.18869 & 45.77 & 0.16756 \\
\hline $51 \mathrm{Peg}$ & 15.47 & 0.053 & 0.013 & 0.472 & 55.16 & 0.18147 & 55.16 & 0.18147 & 54.74 & 0.18074 \\
\hline HIP 79431 & 14.54 & 0.360 & 0.290 & 2.100 & 47.90 & 0.17905 & 56.73 & 0.19474 & 50.35 & 0.17237 \\
\hline tau Boo & 15.66 & 0.049 & 0.011 & 4.320 & 55.17 & 0.17870 & 55.17 & 0.17870 & 55.17 & 0.17870 \\
\hline HD 177565 & 16.93 & 0.246 & 0.059 & 0.048 & 53.47 & 0.16164 & 53.54 & 0.16175 & 32.83 & 0.12114 \\
\hline GJ 3942 & 16.94 & 0.061 & 0.121 & 0.022 & 53.50 & 0.16162 & 53.51 & 0.16162 & 36.21 & 0.12787 \\
\hline GJ 504 & 17.54 & 43.50 & $0.000^{\mathrm{a}}$ & 4.000 & 50.98 & 0.15338 & 50.44 & 0.15253 & 46.06 & 0.14327 \\
\hline $\begin{array}{l}\text { LSPM } \\
\mathrm{J} 2116+0234\end{array}$ & 17.64 & 0.088 & 0.183 & 0.042 & 52.71 & 0.15288 & 52.72 & 0.15289 & 36.69 & 0.12224 \\
\hline
\end{tabular}


Table 5 (continued)

\begin{tabular}{|c|c|c|c|c|c|c|c|c|c|c|}
\hline \multicolumn{2}{|c|}{ Target } & \multicolumn{3}{|c|}{ Known Planet } & \multicolumn{2}{|c|}{ Petrovich's Crit. } & \multicolumn{2}{|c|}{ Giuppone's Crit. } & \multicolumn{2}{|c|}{ Hill AMD Crit. } \\
\hline Name & $\begin{array}{c}\text { Distance } \\
\text { (pc) }\end{array}$ & $\begin{array}{c}a_{k} \\
(\mathrm{AU})\end{array}$ & $e_{k}$ & $\begin{array}{c}m_{k} \sin I \\
\left(M_{\mathrm{J}}\right)\end{array}$ & $\begin{array}{l}\text { DoS } \\
\text { (Dynan }\end{array}$ & $\begin{array}{l}\text { Completeness } \\
\text { nically Stable) }\end{array}$ & $\begin{array}{l}\text { DoS } \\
\text { (Dyna }\end{array}$ & $\begin{array}{l}\text { Completeness } \\
\text { nically Stable) }\end{array}$ & $\begin{array}{l}\text { DoS } \\
\text { (Dyna }\end{array}$ & $\begin{array}{l}\text { Completeness } \\
\text { nically Stable) }\end{array}$ \\
\hline HN Peg & 18.13 & 773.0 & $0.000^{\mathrm{a}}$ & 21.999 & 51.48 & 0.14691 & 51.24 & 0.14632 & 51.48 & 0.14691 \\
\hline HD 99492 & 18.21 & 0.120 & 0.250 & 0.070 & 51.17 & 0.14590 & 51.21 & 0.14596 & 36.75 & 0.11846 \\
\hline 70 Vir & 17.91 & 0.481 & 0.399 & 7.416 & 45.41 & 0.13822 & 51.75 & 0.14952 & 31.19 & 0.06767 \\
\hline WD 0806-661 & 19.20 & 2500 & $0.000^{\mathrm{a}}$ & 7.500 & 50.01 & 0.13453 & 49.77 & 0.13407 & 50.01 & 0.13453 \\
\hline GJ 3021 & 17.56 & 0.490 & 0.511 & 3.370 & 41.06 & 0.13326 & 52.29 & 0.15379 & 31.75 & 0.07325 \\
\hline HR 810 & 17.33 & 0.920 & 0.140 & 2.270 & 39.78 & 0.13240 & 52.06 & 0.15568 & 41.01 & 0.13327 \\
\hline HD 192263 & 19.65 & 0.150 & 0.050 & 0.560 & 49.38 & 0.12922 & 49.39 & 0.12923 & 47.09 & 0.12526 \\
\hline GJ 3634 & 19.80 & 0.029 & 0.080 & $0.026^{\mathrm{b}}$ & 49.36 & 0.12767 & 49.36 & 0.12767 & 36.59 & 0.10460 \\
\hline HD 104067 & 20.38 & 0.260 & 0.000 & 0.160 & 48.34 & 0.12136 & 48.41 & 0.12147 & 33.65 & 0.09502 \\
\hline GJ 649 & 10.38 & 1.135 & 0.300 & 0.328 & 19.05 & 0.11665 & 33.41 & 0.17755 & 22.74 & 0.12044 \\
\hline GJ 4276 & 21.35 & 0.082 & 0.370 & 0.052 & 46.58 & 0.11155 & 46.59 & 0.11157 & 33.42 & 0.08847 \\
\hline HD 27442 & 18.28 & 1.271 & 0.060 & 1.560 & 33.29 & 0.10893 & 49.20 & 0.14089 & 33.73 & 0.11127 \\
\hline HD 90156 & 21.96 & 0.250 & 0.310 & 0.057 & 45.78 & 0.10546 & 45.96 & 0.10574 & 25.83 & 0.07132 \\
\hline HD 4308 & 22.03 & 0.120 & 0.000 & 0.050 & 45.71 & 0.10502 & 45.71 & 0.10502 & 29.56 & 0.07736 \\
\hline HD 147513 & 12.91 & 1.320 & 0.260 & 1.210 & 18.79 & 0.09736 & 36.64 & 0.16028 & 17.04 & 0.07235 \\
\hline GJ 3341 & 23.16 & 0.089 & 0.310 & 0.021 & 44.18 & 0.09466 & 44.18 & 0.09467 & 24.63 & 0.06263 \\
\hline HD 39855 & 23.28 & 0.041 & 0.140 & 0.027 & 43.83 & 0.09364 & 43.83 & 0.09364 & 31.55 & 0.07342 \\
\hline HIP 12961 & 23.39 & 0.250 & 0.170 & 0.360 & 43.40 & 0.09254 & 43.49 & 0.09267 & 36.19 & 0.08064 \\
\hline HD 62509 & 10.34 & 1.640 & 0.020 & 2.300 & 14.18 & 0.08797 & 27.82 & 0.14986 & 23.77 & 0.14131 \\
\hline alf Ari & 20.21 & 1.200 & 0.250 & 1.800 & 29.04 & 0.08582 & 46.20 & 0.11947 & 30.04 & 0.08280 \\
\hline HD 156668 & 24.35 & 0.050 & 0.000 & 0.013 & 41.73 & 0.08465 & 41.73 & 0.08465 & 23.46 & 0.05560 \\
\hline HD 42618 & 24.35 & 0.554 & 0.190 & 0.045 & 40.26 & 0.08238 & 41.72 & 0.08465 & 18.05 & 0.04721 \\
\hline HD 19994 & 22.54 & 1.305 & 0.063 & 1.370 & 31.71 & 0.07743 & 44.26 & 0.09913 & 28.45 & 0.07212 \\
\hline HD 16417 & 25.41 & 0.140 & 0.200 & 0.070 & 40.37 & 0.07640 & 40.37 & 0.07641 & 26.21 & 0.05474 \\
\hline alf Tau & 20.43 & 1.460 & 0.100 & 6.470 & 23.19 & 0.06815 & 44.02 & 0.11328 & 31.05 & 0.08371 \\
\hline HD 103949 & 26.52 & 0.439 & 0.190 & 0.035 & 37.92 & 0.06750 & 38.51 & 0.06837 & 15.20 & 0.03491 \\
\hline HD 33564 & 20.97 & 1.100 & 0.340 & 9.100 & 23.44 & 0.06595 & 45.16 & 0.11161 & 22.33 & 0.04146 \\
\hline HD 210277 & 21.31 & 1.130 & 0.480 & 1.290 & 21.57 & 0.06332 & 42.77 & 0.10527 & 20.89 & 0.04926 \\
\hline HD 179949 & 27.48 & 0.044 & 0.022 & 0.916 & 37.02 & 0.06204 & 37.02 & 0.06204 & 37.01 & 0.06204 \\
\hline gam Cep & 13.54 & 2.050 & 0.049 & 1.850 & 11.63 & 0.05867 & 24.54 & 0.11039 & 19.60 & 0.09681 \\
\hline HD 125595 & 28.22 & 0.080 & 0.000 & 0.020 & 35.59 & 0.05742 & 35.59 & 0.05742 & 18.03 & 0.03351 \\
\hline HD 164595 & 28.28 & 0.230 & 0.088 & 0.051 & 35.64 & 0.05703 & 35.65 & 0.05704 & 16.91 & 0.03172 \\
\hline HD 10647 & 17.34 & 2.015 & 0.150 & 0.940 & 14.44 & 0.05699 & 29.24 & 0.10065 & 19.66 & 0.07537 \\
\hline HD 93083 & 28.54 & 0.477 & 0.140 & 0.370 & 34.77 & 0.05476 & 35.31 & 0.05554 & 23.95 & 0.03989 \\
\hline HD 75289 & 29.14 & 0.050 & 0.030 & 0.490 & 34.48 & 0.05209 & 34.48 & 0.05209 & 34.42 & 0.05201 \\
\hline HD 21411 & 29.16 & 0.362 & 0.400 & 0.207 & 34.03 & 0.05142 & 34.43 & 0.05199 & 21.75 & 0.03520 \\
\hline $\begin{array}{l}\text { HD } 102195 \\
\text { DENIS-P }\end{array}$ & 29.36 & 0.050 & 0.000 & 0.410 & 33.97 & 0.05095 & 33.97 & 0.05095 & 33.87 & 0.05082 \\
\hline $\begin{array}{l}\text { J082303.1- } \\
491201\end{array}$ & 20.77 & 0.360 & 0.345 & $28.500^{\mathrm{b}}$ & 17.12 & 0.05088 & 47.51 & 0.11737 & 21.82 & 0.03981 \\
\hline
\end{tabular}


Table 5 (continued)

\begin{tabular}{|c|c|c|c|c|c|c|c|c|c|c|}
\hline \multicolumn{2}{|c|}{ Target } & \multicolumn{3}{|c|}{ Known Planet } & \multicolumn{2}{|c|}{ Petrovich's Crit. } & \multicolumn{2}{|c|}{ Giuppone's Crit. } & \multicolumn{2}{|c|}{ Hill AMD Crit. } \\
\hline Name & $\begin{array}{c}\text { Distance } \\
\text { (pc) }\end{array}$ & $\begin{array}{c}a_{k} \\
(\mathrm{AU})\end{array}$ & $e_{k}$ & $\begin{array}{c}m_{k} \sin I \\
\left(M_{\mathrm{J}}\right)\end{array}$ & $\begin{array}{l}\text { DoS } \\
\text { (Dyna }\end{array}$ & $\begin{array}{l}\text { Completeness } \\
\text { mically Stable) }\end{array}$ & $\begin{array}{l}\text { DoS } \\
\text { (Dyna }\end{array}$ & $\begin{array}{l}\text { Completeness } \\
\text { aically Stable) }\end{array}$ & $\begin{array}{l}\text { DoS } \\
\text { (Dyna) }\end{array}$ & $\begin{array}{l}\text { ompleteness } \\
\text { cally Stable) }\end{array}$ \\
\hline GJ 3470 & 29.45 & 0.036 & $0.000^{\mathrm{a}}$ & $0.044^{\mathrm{b}}$ & 33.75 & 0.05047 & 33.75 & 0.05047 & 26.41 & 0.04063 \\
\hline HD 46375 & 29.58 & 0.040 & 0.063 & 0.226 & 33.59 & 0.04972 & 33.59 & 0.04972 & 33.32 & 0.04935 \\
\hline HD 101930 & 30.05 & 0.300 & 0.110 & 0.250 & 32.88 & 0.04722 & 32.94 & 0.04731 & 23.67 & 0.03529 \\
\hline HD 52265 & 30.01 & 0.520 & 0.270 & 1.210 & 32.53 & 0.04681 & 33.04 & 0.04751 & 29.59 & 0.04284 \\
\hline HD 218566 & 28.85 & 0.690 & 0.300 & 0.200 & 29.75 & 0.04656 & 34.74 & 0.05377 & 16.42 & 0.02951 \\
\hline $7 \mathrm{CMa}$ & 19.82 & 1.930 & 0.220 & 2.460 & 13.86 & 0.04475 & 31.99 & 0.09056 & 15.12 & 0.04120 \\
\hline HD 162020 & 30.85 & 0.080 & 0.280 & 9.840 & 31.45 & 0.04335 & 31.45 & 0.04335 & 31.40 & 0.04323 \\
\hline HD 8326 & 30.71 & 0.533 & 0.200 & 0.210 & 31.06 & 0.04303 & 31.78 & 0.04402 & 16.70 & 0.02539 \\
\hline HD 128356 & 26.03 & 0.870 & 0.570 & 0.890 & 19.22 & 0.04017 & 38.84 & 0.07122 & 24.53 & 0.04694 \\
\hline HD 64114 & 31.55 & 0.246 & 0.120 & 0.056 & 30.40 & 0.04010 & 30.41 & 0.04011 & 13.48 & 0.02021 \\
\hline HD 130322 & 31.91 & 0.093 & 0.029 & 1.150 & 29.96 & 0.03848 & 29.96 & 0.03849 & 29.94 & 0.03846 \\
\hline HIP 71135 & 32.36 & 0.335 & 0.210 & 0.059 & 28.97 & 0.03642 & 29.08 & 0.03656 & 11.31 & 0.01658 \\
\hline HIP 35173 & 33.19 & 0.217 & 0.160 & 0.040 & 27.96 & 0.03323 & 27.97 & 0.03324 & 11.01 & 0.01494 \\
\hline mu Leo & 32.63 & 1.100 & 0.090 & 2.400 & 26.48 & 0.03232 & 28.86 & 0.03546 & 23.87 & 0.02925 \\
\hline HD 40979 & 34.12 & 0.850 & 0.250 & 4.670 & 24.83 & 0.02778 & 26.47 & 0.02987 & 25.52 & 0.02862 \\
\hline HD 45652 & 34.89 & 0.237 & 0.607 & 0.433 & 25.25 & 0.02719 & 25.32 & 0.02727 & 22.62 & 0.02423 \\
\hline HD 63765 & 32.57 & 0.940 & 0.240 & 0.530 & 20.81 & 0.02498 & 28.98 & 0.03568 & 15.36 & 0.01996 \\
\hline BD-11 4672 & 27.30 & 2.280 & 0.050 & 0.530 & 11.52 & 0.02441 & 28.86 & 0.04998 & 12.48 & 0.02800 \\
\hline 16 Cyg B & 21.15 & 1.660 & 0.680 & 1.780 & 7.44 & 0.02285 & 24.33 & 0.06426 & 5.34 & 0.00867 \\
\hline HD 114386 & 27.95 & 1.730 & 0.230 & 1.140 & 11.42 & 0.02260 & 31.50 & 0.05179 & 15.49 & 0.02924 \\
\hline HD 216770 & 36.70 & 0.460 & 0.370 & 0.570 & 22.18 & 0.02132 & 22.65 & 0.02187 & 17.91 & 0.01700 \\
\hline HD 195019 & 37.71 & 0.140 & 0.010 & 3.980 & 21.21 & 0.01928 & 21.21 & 0.01928 & 21.21 & 0.01928 \\
\hline HD 63454 & 37.73 & 0.040 & 0.000 & 0.250 & 21.17 & 0.01923 & 21.17 & 0.01923 & 21.08 & 0.01913 \\
\hline HD 117618 & 37.82 & 0.180 & 0.150 & 0.174 & 20.98 & 0.01899 & 20.99 & 0.01899 & 16.18 & 0.01438 \\
\hline HD 16141 & 37.83 & 0.360 & 0.250 & 0.260 & 20.94 & 0.01894 & 20.97 & 0.01897 & 14.07 & 0.01248 \\
\hline HD 23079 & 33.49 & 1.600 & 0.100 & 2.610 & 16.96 & 0.01861 & 27.25 & 0.03183 & 18.28 & 0.02111 \\
\hline HD 111998 & 33.26 & 1.820 & 0.030 & 4.510 & 16.75 & 0.01858 & 27.42 & 0.03244 & 19.26 & 0.02234 \\
\hline HD 113337 & 36.22 & 0.920 & 0.460 & 2.830 & 18.71 & 0.01770 & 23.32 & 0.02320 & 21.01 & 0.02045 \\
\hline $\begin{array}{l}\text { HD } 108147 \\
\text { 2MASS }\end{array}$ & 38.96 & 0.102 & 0.530 & 0.261 & 19.41 & 0.01644 & 19.41 & 0.01644 & 18.53 & 0.01557 \\
\hline $\begin{array}{l}\text { J01225093- } \\
2439505\end{array}$ & 36.00 & 52.00 & $0.000^{\mathrm{a}}$ & 24.500 & 12.10 & 0.01626 & 2.54 & 0.00413 & 12.56 & 0.01697 \\
\hline HD 4208 & 34.23 & 1.662 & 0.042 & 0.810 & 15.58 & 0.01615 & 26.02 & 0.02920 & 10.49 & 0.01245 \\
\hline $\begin{array}{l}\text { HD } 102117 \\
\text { 2MASS }\end{array}$ & 39.62 & 0.150 & 0.120 & 0.170 & 18.57 & 0.01508 & 18.57 & 0.01508 & 15.14 & 0.01200 \\
\hline $\begin{array}{l}\text { J02192210- } \\
3925225\end{array}$ & 39.40 & 156.0 & $0.000^{\mathrm{a}}$ & 13.900 & 16.03 & 0.01491 & 1.07 & 0.00145 & 13.59 & 0.01395 \\
\hline GJ 849 & 8.80 & 2.350 & 0.040 & 1.000 & 2.11 & 0.01476 & 6.51 & 0.04162 & 8.14 & 0.04811 \\
\hline HD 28185 & 39.43 & 1.020 & 0.050 & 5.900 & 18.00 & 0.01449 & 18.89 & 0.01545 & 18.26 & 0.01480 \\
\hline gam 1 Leo & 38.52 & 1.190 & 0.144 & 8.780 & 17.35 & 0.01446 & 19.94 & 0.01736 & 19.33 & 0.01668 \\
\hline HD 114762 & 40.23 & 0.360 & 0.340 & 10.690 & 17.68 & 0.01384 & 17.74 & 0.01391 & 17.70 & 0.01385 \\
\hline
\end{tabular}


Table 5 (continued)

\begin{tabular}{|c|c|c|c|c|c|c|c|c|c|c|}
\hline \multicolumn{2}{|c|}{ Target } & \multicolumn{3}{|c|}{ Known Planet } & \multicolumn{2}{|c|}{ Petrovich's Crit. } & \multicolumn{2}{|c|}{ Giuppone's Crit. } & \multicolumn{2}{|c|}{ Hill AMD Crit. } \\
\hline Name & $\begin{array}{c}\text { Distance } \\
\text { (pc) }\end{array}$ & $\begin{array}{c}a_{k} \\
(\mathrm{AU})\end{array}$ & $e_{k}$ & $\begin{array}{c}m_{k} \sin I \\
\left(M_{\mathrm{J}}\right)\end{array}$ & $\begin{array}{l}\text { DoS } \\
\text { (Dyna }\end{array}$ & $\begin{array}{l}\text { Completeness } \\
\text { mically Stable) }\end{array}$ & $\begin{array}{l}\text { DoS } \\
\text { (Dyna }\end{array}$ & $\begin{array}{l}\text { Completeness } \\
\text { aically Stable) }\end{array}$ & $\begin{array}{l}\text { DoS } \\
\text { (Dyna }\end{array}$ & $\begin{array}{l}\text { Completeness } \\
\text { ically Stable) }\end{array}$ \\
\hline HD 38283 & 38.10 & 1.020 & 0.410 & 0.400 & 16.19 & 0.01338 & 20.61 & 0.01833 & 8.88 & 0.00781 \\
\hline HD 111232 & 28.98 & 1.970 & 0.200 & 7.140 & 8.24 & 0.01314 & 28.55 & 0.04394 & 15.50 & 0.02016 \\
\hline HD 142415 & 35.57 & 1.060 & 0.500 & 1.670 & 13.83 & 0.01269 & 24.28 & 0.02504 & 18.26 & 0.01827 \\
\hline HD 83443 & 40.95 & 0.040 & 0.010 & 0.340 & 16.85 & 0.01262 & 16.85 & 0.01262 & 16.83 & 0.01261 \\
\hline HD 178911 B & 41.02 & 0.340 & 0.110 & 8.030 & 16.73 & 0.01250 & 16.73 & 0.01251 & 16.73 & 0.01250 \\
\hline HD 98736 & 32.48 & 1.864 & 0.226 & 2.330 & 9.52 & 0.01187 & 26.59 & 0.03299 & 14.86 & 0.01855 \\
\hline kap CrB & 30.09 & 2.650 & 0.167 & 1.811 & 7.73 & 0.01176 & 23.13 & 0.03227 & 11.24 & 0.01784 \\
\hline HD 89744 & 38.68 & 0.917 & 0.677 & 8.350 & 14.97 & 0.01165 & 19.88 & 0.01700 & 18.04 & 0.01450 \\
\hline HD 103720 & 41.60 & 0.050 & 0.086 & 0.620 & 16.08 & 0.01155 & 16.08 & 0.01155 & 16.08 & 0.01155 \\
\hline bet UMi & 38.78 & 1.400 & 0.190 & 6.100 & 14.89 & 0.01153 & 19.69 & 0.01677 & 17.97 & 0.01495 \\
\hline HD 7199 & 36.19 & 1.360 & 0.190 & 0.270 & 13.21 & 0.01152 & 23.30 & 0.02319 & 6.47 & 0.00720 \\
\hline HD 168746 & 41.62 & 0.070 & 0.110 & 0.270 & 16.05 & 0.01152 & 16.05 & 0.01152 & 15.81 & 0.01130 \\
\hline HD 121504 & 41.71 & 0.330 & 0.030 & 1.510 & 15.91 & 0.01138 & 15.91 & 0.01138 & 15.69 & 0.01117 \\
\hline HD 10697 & 33.15 & 2.140 & 0.104 & 6.383 & 10.25 & 0.01096 & 25.68 & 0.03042 & 16.91 & 0.01923 \\
\hline HD 197037 & 33.00 & 2.070 & 0.220 & 0.790 & 8.95 & 0.01082 & 25.09 & 0.02977 & 9.41 & 0.01284 \\
\hline HD 216437 & 26.71 & 2.497 & 0.317 & 2.223 & 5.26 & 0.01078 & 20.39 & 0.03537 & 7.52 & 0.01112 \\
\hline HD 216435 & 33.01 & 2.560 & 0.070 & 1.260 & 9.27 & 0.01068 & 23.88 & 0.02806 & 9.03 & 0.01220 \\
\hline HD 6434 & 42.41 & 0.140 & 0.170 & 0.490 & 15.02 & 0.01032 & 15.02 & 0.01032 & 14.71 & 0.01005 \\
\hline HD 204941 & 28.74 & 2.550 & 0.370 & 0.230 & 4.86 & 0.01021 & 16.93 & 0.02538 & 7.84 & 0.01662 \\
\hline GJ 179 & 12.36 & 2.410 & 0.210 & 0.820 & 1.91 & 0.01016 & 6.70 & 0.03552 & 3.80 & 0.01860 \\
\hline HD 85390 & 33.56 & 1.373 & 0.500 & 0.099 & 8.04 & 0.00978 & 26.31 & 0.03052 & 6.17 & 0.00962 \\
\hline HD 141937 & 33.39 & 1.500 & 0.410 & 9.690 & 9.38 & 0.00970 & 26.47 & 0.03104 & 19.33 & 0.01926 \\
\hline HD 49674 & 43.09 & 0.060 & 0.090 & 0.100 & 14.26 & 0.00937 & 14.26 & 0.00937 & 12.93 & 0.00832 \\
\hline HD 137388 & 40.53 & 0.890 & 0.360 & 0.200 & 13.01 & 0.00899 & 17.31 & 0.01333 & 5.17 & 0.00387 \\
\hline HD 126525 & 37.69 & 1.837 & 0.035 & 0.237 & 11.74 & 0.00896 & 20.96 & 0.01911 & 3.96 & 0.00421 \\
\hline iot Dra & 31.67 & 1.275 & 0.712 & 8.820 & 6.90 & 0.00877 & 28.10 & 0.03667 & 20.24 & 0.02221 \\
\hline HD 208487 & 44.00 & 0.524 & 0.240 & 0.520 & 13.22 & 0.00816 & 13.27 & 0.00820 & 9.89 & 0.00578 \\
\hline $91 \mathrm{Aqr}$ & 44.08 & 0.700 & 0.027 & 3.200 & 13.14 & 0.00808 & 13.17 & 0.00811 & 12.88 & 0.00787 \\
\hline HD 42012 & 36.84 & 1.670 & 0.200 & 1.600 & 9.16 & 0.00714 & 22.02 & 0.02104 & 11.56 & 0.01050 \\
\hline HD 285507 & 45.09 & 0.060 & 0.090 & 0.980 & 12.10 & 0.00699 & 12.10 & 0.00699 & 12.10 & 0.00699 \\
\hline HD 8574 & 44.88 & 0.760 & 0.300 & 2.030 & 12.04 & 0.00698 & 12.31 & 0.00721 & 11.44 & 0.00654 \\
\hline 30 Ari B & 44.71 & 0.990 & 0.290 & 13.820 & 12.03 & 0.00696 & 12.52 & 0.00739 & 12.48 & 0.00735 \\
\hline HD 330075 & 45.36 & 0.040 & 0.000 & 0.480 & 11.82 & 0.00670 & 11.82 & 0.00670 & 11.82 & 0.00670 \\
\hline HIP 91258 & 45.95 & 0.060 & 0.020 & 1.090 & 11.20 & 0.00612 & 11.20 & 0.00612 & 11.19 & 0.00612 \\
\hline HD 77338 & 46.00 & 0.060 & 0.090 & 0.060 & 11.14 & 0.00608 & 11.14 & 0.00608 & 8.95 & 0.00468 \\
\hline HD 114729 & 37.85 & 2.067 & 0.079 & 0.825 & 8.61 & 0.00602 & 20.36 & 0.01824 & 6.17 & 0.00546 \\
\hline HD 17674 & 44.48 & 1.420 & 0.130 & 0.870 & 9.90 & 0.00523 & 12.70 & 0.00765 & 6.06 & 0.00317 \\
\hline HD 29021 & 31.02 & 2.280 & 0.459 & 2.400 & 3.11 & 0.00522 & 18.51 & 0.02335 & 6.64 & 0.00591 \\
\hline BD-17 63 & 34.49 & 1.340 & 0.540 & 2.850 & 3.37 & 0.00520 & 23.81 & 0.02595 & 12.99 & 0.01150 \\
\hline
\end{tabular}


Table 5 (continued)

\begin{tabular}{|c|c|c|c|c|c|c|c|c|c|c|}
\hline \multicolumn{2}{|c|}{ Target } & \multicolumn{3}{|c|}{ Known Planet } & \multicolumn{2}{|c|}{ Petrovich's Crit. } & \multicolumn{2}{|c|}{ Giuppone's Crit. } & \multicolumn{2}{|c|}{ Hill AMD Crit. } \\
\hline Name & $\begin{array}{c}\text { Distance } \\
\text { (pc) }\end{array}$ & $\begin{array}{c}a_{k} \\
(\mathrm{AU})\end{array}$ & $e_{k}$ & $\begin{array}{c}m_{k} \sin I \\
\left(M_{\mathrm{J}}\right)\end{array}$ & $\begin{array}{l}\text { DoS } \\
\text { (Dyna }\end{array}$ & $\begin{array}{l}\text { Completeness } \\
\text { mically Stable) }\end{array}$ & $\begin{array}{l}\text { DoS } \\
\text { (Dyna }\end{array}$ & $\begin{array}{l}\text { Completeness } \\
\text { aically Stable) }\end{array}$ & $\begin{array}{l}\text { DoS } \\
\text { (Dyna) }\end{array}$ & $\begin{array}{l}\text { ompleteness } \\
\text { cally Stable) }\end{array}$ \\
\hline HD 70642 & 29.30 & 3.318 & 0.175 & 1.993 & 2.99 & 0.00505 & 14.53 & 0.01938 & 6.06 & 0.00960 \\
\hline HD 164604 & 39.41 & 1.331 & 0.350 & 1.998 & 8.13 & 0.00496 & 18.74 & 0.01538 & 13.10 & 0.01006 \\
\hline HD 210193 & 42.25 & 1.487 & 0.240 & 0.482 & 9.03 & 0.00483 & 15.22 & 0.01051 & 4.79 & 0.00296 \\
\hline HD 30562 & 26.18 & 2.340 & 0.760 & 1.220 & 2.38 & 0.00482 & 12.25 & 0.02060 & 2.37 & 0.00213 \\
\hline HD 22781 & 32.63 & 1.167 & 0.819 & 13.650 & 3.97 & 0.00449 & 26.98 & 0.03284 & 20.10 & 0.02080 \\
\hline GU Psc & 48.00 & 2000 & $0.000^{\mathrm{a}}$ & 11.300 & 9.27 & 0.00442 & 0.00 & 0.00000 & 9.27 & 0.00442 \\
\hline HR 2562 & 34.04 & 20.30 & $0.000^{\mathrm{a}}$ & 30.000 & 2.55 & 0.00439 & 2.02 & 0.00404 & 5.38 & 0.00931 \\
\hline HD 154345 & 18.29 & 4.210 & 0.040 & 0.820 & 1.43 & 0.00423 & 5.21 & 0.01524 & 4.91 & 0.01739 \\
\hline HD 20782 & 36.02 & 1.365 & 0.950 & 1.488 & 3.35 & 0.00401 & 21.26 & 0.02067 & 10.49 & 0.00813 \\
\hline HD 143105 & 48.70 & 0.038 & 0.070 & 1.210 & 8.63 & 0.00394 & 8.63 & 0.00394 & 8.63 & 0.00394 \\
\hline HD 89307 & 32.04 & 3.270 & 0.200 & 2.110 & 2.68 & 0.00373 & 14.68 & 0.01598 & 5.72 & 0.00700 \\
\hline HD 107148 & 49.49 & 0.269 & 0.050 & 0.210 & 8.01 & 0.00344 & 8.01 & 0.00344 & 5.79 & 0.00233 \\
\hline HD 196885 & 34.20 & 2.370 & 0.480 & 2.580 & 2.63 & 0.00330 & 17.08 & 0.01708 & 7.36 & 0.00542 \\
\hline WASP-80 & 49.86 & 0.034 & 0.002 & $0.538^{\mathrm{b}}$ & 7.69 & 0.00323 & 7.69 & 0.00323 & 7.69 & 0.00323 \\
\hline HD 167042 & 49.73 & 1.320 & 0.089 & 1.700 & 7.46 & 0.00307 & 7.81 & 0.00330 & 5.84 & 0.00226 \\
\hline $\mathrm{BD}+144559$ & 49.42 & 0.780 & 0.290 & 1.040 & 7.16 & 0.00288 & 8.07 & 0.00348 & 6.38 & 0.00257 \\
\hline HD 50554 & 31.19 & 2.353 & 0.501 & 4.954 & 1.97 & 0.00283 & 16.95 & 0.02027 & 7.53 & 0.00593 \\
\hline HD 81040 & 34.47 & 1.940 & 0.530 & 7.270 & 2.71 & 0.00281 & 20.06 & 0.02082 & 12.91 & 0.01062 \\
\hline eps Tau & 49.23 & 1.930 & 0.151 & 7.600 & 6.89 & 0.00270 & 8.17 & 0.00359 & 7.48 & 0.00315 \\
\hline HD 153950 & 48.52 & 1.280 & 0.340 & 2.950 & 6.84 & 0.00269 & 8.81 & 0.00406 & 7.66 & 0.00335 \\
\hline HD 100777 & 49.60 & 1.030 & 0.360 & 1.030 & 6.42 & 0.00241 & 7.90 & 0.00338 & 5.49 & 0.00213 \\
\hline HD 117207 & 32.38 & 3.787 & 0.157 & 1.926 & 1.63 & 0.00223 & 10.90 & 0.01051 & 4.04 & 0.00535 \\
\hline HD 213240 & 40.92 & 1.890 & 0.420 & 5.580 & 4.87 & 0.00213 & 16.16 & 0.01187 & 12.61 & 0.00835 \\
\hline HD 32963 & 38.12 & 3.410 & 0.070 & 0.700 & 2.16 & 0.00203 & 13.41 & 0.00986 & 2.56 & 0.00270 \\
\hline 14 Her & 17.94 & 2.930 & 0.370 & 4.660 & 0.55 & 0.00136 & 5.33 & 0.01550 & 0.80 & 0.00067 \\
\hline HD 222582 & 42.21 & 1.340 & 0.730 & 8.370 & 3.38 & 0.00117 & 15.06 & 0.01034 & 13.88 & 0.00912 \\
\hline HD 156846 & 47.80 & 1.120 & 0.850 & 10.670 & 3.93 & 0.00103 & 9.42 & 0.00456 & 9.32 & 0.00448 \\
\hline HD 187085 & 45.96 & 2.100 & 0.251 & 0.836 & 3.53 & 0.00086 & 10.95 & 0.00591 & 3.24 & 0.00137 \\
\hline HD 4113 & 41.92 & 1.280 & 0.903 & 1.560 & 1.94 & 0.00081 & 15.17 & 0.01060 & 9.63 & 0.00589 \\
\hline HD 7449 & 38.71 & 2.380 & 0.920 & 0.508 & 0.66 & 0.00075 & 8.76 & 0.00494 & 1.65 & 0.00073 \\
\hline HD 70573 & 45.70 & 1.760 & 0.400 & 6.100 & 2.91 & 0.00063 & 11.27 & 0.00620 & 9.58 & 0.00491 \\
\hline eps Eri & 3.21 & 3.390 & 0.702 & $1.550^{\mathrm{b}}$ & 0.09 & 0.00046 & 7.00 & 0.04020 & 0.13 & 0.00016 \\
\hline HD 106252 & 38.23 & 2.610 & 0.480 & 6.930 & 0.52 & 0.00044 & 11.99 & 0.00814 & 7.11 & 0.00350 \\
\hline HD $142022 \mathrm{~A}$ & 34.31 & 2.930 & 0.530 & 4.440 & 0.43 & 0.00044 & 9.28 & 0.00700 & 3.70 & 0.00157 \\
\hline HD 171238 & 44.87 & 2.570 & 0.234 & 2.720 & 1.30 & 0.00021 & 11.00 & 0.00598 & 4.65 & 0.00185 \\
\hline HD 86226 & 45.74 & 2.840 & 0.150 & 0.920 & 1.52 & 0.00020 & 10.21 & 0.00525 & 1.81 & 0.00060 \\
\hline HD 87883 & 18.30 & 3.580 & 0.530 & 1.540 & 0.13 & 0.00019 & 1.56 & 0.00300 & 0.16 & 0.00008 \\
\hline psi 1 Dra B & 22.16 & 4.430 & 0.400 & 1.530 & 0.13 & 0.00016 & 1.67 & 0.00217 & 0.26 & 0.00017 \\
\hline HD 20868 & 47.79 & 0.950 & 0.750 & 1.250 & 1.35 & 0.00015 & 9.44 & 0.00457 & 6.91 & 0.00304 \\
\hline
\end{tabular}


Table 5 (continued)

\begin{tabular}{|c|c|c|c|c|c|c|c|c|c|c|}
\hline \multicolumn{2}{|c|}{ Target } & \multicolumn{3}{|c|}{ Known Planet } & \multicolumn{2}{|c|}{ Petrovich's Crit. } & \multicolumn{2}{|c|}{ Giuppone's Crit. } & \multicolumn{2}{|c|}{ Hill AMD Crit. } \\
\hline Name & $\begin{array}{c}\text { Distance } \\
\text { (pc) }\end{array}$ & $\begin{array}{c}a_{k} \\
(\mathrm{AU})\end{array}$ & $e_{k}$ & $\begin{array}{c}m_{k} \sin I \\
\left(M_{\mathrm{J}}\right)\end{array}$ & $\begin{array}{l}\text { DoS } \\
\text { (Dyna }\end{array}$ & $\begin{array}{l}\text { Completeness } \\
\text { nically Stable) }\end{array}$ & $\begin{array}{l}\text { DoS } \\
\text { (Dyna }\end{array}$ & $\begin{array}{l}\text { Completeness } \\
\text { nically Stable) }\end{array}$ & $\begin{array}{l}\text { DoS } \\
\text { (Dyna }\end{array}$ & $\begin{array}{l}\text { Completeness } \\
\text { nically Stable) }\end{array}$ \\
\hline RR Cae & 21.20 & 5.300 & 0.000 & 4.200 & 0.11 & 0.00013 & 5.20 & 0.00761 & 0.71 & 0.00133 \\
\hline HD 221420 & 31.17 & 18.50 & 0.420 & 9.700 & 0.04 & 0.00009 & 1.12 & 0.00239 & 0.23 & 0.00035 \\
\hline HD 220689 & 46.94 & 3.396 & 0.054 & 1.118 & 1.04 & 0.00008 & 8.62 & 0.00387 & 1.10 & 0.00023 \\
\hline GJ 328 & 20.54 & 4.500 & 0.370 & 2.300 & 0.03 & 0.00003 & 0.99 & 0.00120 & 0.08 & 0.00003 \\
\hline HD 114613 & 20.29 & 5.340 & 0.458 & 0.357 & 0.02 & 0.00002 & 0.35 & 0.00031 & 0.14 & 0.00016 \\
\hline HD 45350 & 46.94 & 1.920 & 0.778 & 1.790 & 0.15 & 0.00001 & 9.00 & 0.00418 & 4.85 & 0.00192 \\
\hline HD 8673 & 37.90 & 3.020 & 0.723 & 14.200 & 0.01 & 0.00001 & 6.00 & 0.00253 & 4.67 & 0.00144 \\
\hline HD 24040 & 46.68 & 4.920 & 0.040 & 3.860 & 0.05 & 0.00000 & 4.53 & 0.00110 & 0.46 & 0.00003 \\
\hline DE CVn & 30.55 & 5.750 & 0.000 & $12.029^{\mathrm{b}}$ & 0.00 & 0.00000 & 2.40 & 0.00150 & 0.08 & 0.00005 \\
\hline HD 13931 & 47.46 & 5.150 & 0.020 & 2.200 & 0.07 & 0.00000 & 4.32 & 0.00100 & 0.30 & 0.00001 \\
\hline HD 108341 & 49.40 & 2.000 & 0.850 & 3.500 & 0.01 & 0.00000 & 6.66 & 0.00247 & 4.67 & 0.00152 \\
\hline $\begin{array}{l}\text { HD } 79498 \\
\text { CFBDSIR }\end{array}$ & 49.02 & 2.980 & 0.575 & 1.340 & 0.00 & 0.00000 & 4.94 & 0.00134 & 1.46 & 0.00031 \\
\hline $\begin{array}{l}\text { J145829 } \\
+101343\end{array}$ & 23.10 & 2.600 & $0.000^{\mathrm{a}}$ & 10.500 & 0.00 & 0.00000 & 5.64 & 0.01107 & 0.00 & 0.00000 \\
\hline HD 106515 A & 34.12 & 4.590 & 0.572 & 9.610 & 0.00 & 0.00000 & 0.78 & 0.00014 & 0.26 & 0.00001 \\
\hline HIP 70849 & 24.07 & 20.25 & 0.715 & 9.000 & 0.00 & 0.00000 & 0.00 & 0.00000 & 0.00 & 0.00000 \\
\hline HD 150706 & 28.29 & 6.700 & 0.380 & 2.710 & 0.00 & 0.00000 & 0.06 & 0.00001 & 0.00 & 0.00000 \\
\hline HD 219077 & 29.21 & 6.220 & 0.770 & 10.390 & 0.00 & 0.00000 & 0.00 & 0.00000 & 0.00 & 0.00000 \\
\hline HD 25015 & 37.47 & 6.190 & 0.390 & 4.480 & 0.00 & 0.00000 & 0.13 & 0.00001 & 0.00 & 0.00000 \\
\hline HD 196067 & 39.98 & 5.020 & 0.660 & 6.900 & 0.00 & 0.00000 & 0.34 & 0.00002 & 0.18 & 0.00000 \\
\hline HD 98649 & 42.22 & 6.570 & 0.860 & 6.790 & 0.00 & 0.00000 & 0.00 & 0.00000 & 0.00 & 0.00000 \\
\hline HD 13724 & 43.52 & 12.40 & 0.340 & 26.770 & 0.00 & 0.00000 & 0.00 & 0.00000 & 0.00 & 0.00000 \\
\hline HD 92987 & 43.59 & 9.620 & 0.210 & 16.880 & 0.00 & 0.00000 & 0.00 & 0.00000 & 0.00 & 0.00000 \\
\hline HD 166724 & 45.19 & 5.420 & 0.734 & 3.530 & 0.00 & 0.00000 & 0.03 & 0.00000 & 0.00 & 0.00000 \\
\hline HD 133131 B & 47.00 & 6.150 & 0.610 & 2.500 & 0.00 & 0.00000 & 0.02 & 0.00000 & 0.00 & 0.00000 \\
\hline HD 181234 & 47.81 & 7.520 & 0.730 & 8.370 & 0.00 & 0.00000 & 0.00 & 0.00000 & 0.00 & 0.00000 \\
\hline $\begin{array}{l}\text { HD } 220773 \\
\text { WISEP }\end{array}$ & 49.00 & 4.940 & 0.510 & 1.450 & 0.00 & 0.00000 & 0.86 & 0.00003 & 0.14 & 0.00000 \\
\hline $\begin{array}{l}J 121756.91 \\
+162640.2 \mathrm{~A}\end{array}$ & 10.10 & 8.000 & $0.000^{\mathrm{a}}$ & 22.000 & 0.00 & 0.00000 & 0.00 & 0.00000 & 0.00 & 0.00000 \\
\hline
\end{tabular}

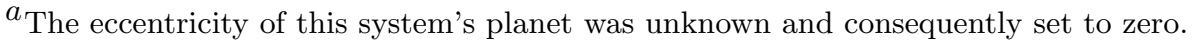

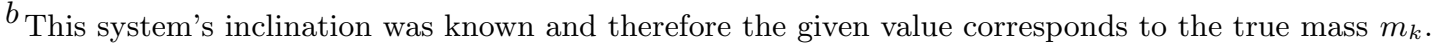

Note-Results are obtained for 213 single-planet systems using Petrovich's, Hill AMD and Giuppone's criteria. In particular, the systems are ranked according to the completeness values calculated with Petrovich's criterion. 